TRANSACTIONS OF THE

AMERICAN MATHEMATICAL SOCIETY

Volume 352, Number 11, Pages 5179-5211

S 0002-9947(00)02443-0

Article electronically published on June 27, 2000

\title{
CENTER MANIFOLDS FOR SMOOTH INVARIANT MANIFOLDS
}

\author{
SHUI-NEE CHOW, WEISHI LIU, AND YINGFEI YI
}

\begin{abstract}
We study dynamics of flows generated by smooth vector fields in $\mathbb{R}^{n}$ in the vicinity of an invariant and closed smooth manifold $Y$. By applying the Hadamard graph transform technique, we show that there exists an invariant manifold (called a center manifold of $Y$ ) based on the information of the linearization along $Y$, which contains every locally bounded solution and is persistent under small perturbations.
\end{abstract}

\section{INTRODUCTION}

This paper is devoted to the study of existence and smoothness of a local center manifold for invariant manifolds of flows. By extending the classical center manifold theory, our primary goal is to build up a geometrical foundation which allows one to study dynamics of a differential equation in the vicinity of an invariant set $Y$ (e.g. a torus or a heteroclinic cycle) in addition to an equilibrium point or a periodic orbit.

The classical center manifolds theory of equilibria, since first introduced by Pliss (39]) and Kelley ([30]) in the 1960's and later developed by many others (e.g. [7, [26], [48], [51], [53] etc.), has become an important subject and found tremendous applications in the study of flows and diffeomorphisms (see [7, 11], 12], 17], [21], 24] and references therein). Besides generalizations to various cases of infinite dimensional semiflows (e.g. [3], [4], [8], [13], [25], 35], [52]), there have been several important extensions of the classical center manifolds theory in the case of invariant sets. Center manifolds along a trajectory of a diffeomorphism were constructed in [26] and [47]. In [19], as part of the geometric theory of singularly perturbed ordinary differential equations, Fenichel showed the existence of center manifolds for invariant manifolds consisting of equilibria (see also 29], [32 for more applications of the theory). Related to perturbation and bifurcation problems, some cases of center manifolds for invariant tori were studied in Chenciner and Iooss ([10]), Chow and $\mathrm{Lu}([14])$. Center manifolds for skew-product flows were studied in Chow and Yi (15]). Recently, Homburg ([27]) and Sandstede ([45]) constructed center manifolds for certain homoclinic orbits to study various global bifurcation problems.

We shall show in this paper that, if a smooth flow in $\mathbb{R}^{n}$ admits a connected, invariant and closed (i.e., compact without boundary) smooth manifold $Y$, then under certain exponential rate conditions on an invariant splitting of the linearization along $Y$, the flow has a smooth locally invariant manifold $M_{c}(Y)$ (a center manifold of $Y$ ) corresponding to the splitting, which contains $Y$ and all locally

Received by the editors June 24, 1996 and, in revised form, March 20, 1998.

1991 Mathematics Subject Classification. Primary 34C30, 34C35, 34D35.

Key words and phrases. Center manifold, graph transform, overflowing.

(C)2000 American Mathematical Society 
bounded solutions, and is persistent under small perturbations (see Section 2 for details).

The center manifolds theory we shall present is also closely related to the theory of normally hyperbolic invariant manifolds - a subject which has been extensively studied (see [16, [18], [26], 31], 34], 42], 55] and references therein). In the 1970's, Sacker (42]), and Fenichel ([18]) showed that an $r$-normally hyperbolic overflowing compact invariant $C^{r}$ manifold $Y$ of a flow is $C^{r}$ persistent, and the invariant manifold obtained after perturbation is unique (see [26] for parallel results for diffeomorphisms, [5], [32] for infinite dimensional semiflows, and [23], 25], [44], [56] for non-autonomous systems). Later, it was shown by Mañé (34]), and by Bronstein and Kopanskii ([6]) that, if $Y$ is a $C^{r}$ invariant closed manifold of a $C^{r}$ flow, then $r$-normal hyperbolicity is equivalent to $C^{r}$ persistence and isolation. Recently, Pliss and Sell ([40]) introduced the concept of a weakly, normally hyperbolic invariant set and showed persistence results for such a set.

Our result can be viewed as a generalization to both the theory of normally hyperbolic invariant manifolds and the center manifolds theory of Fenichel ([19]) and Chenciner and Iooss ([10]). Related to the former theory, our work simply provides information when normal hyperbolicity fails. Comparing with [19] in which the invariant manifold consists of equilibria and with [10] in which a subsystem of the linearization along the invariant torus is independent of the points on the torus, our center manifolds theory deals with general flows on an invariant manifold, and the 'center bundle' associated to the linearization along the invariant manifold need not have a constant structure.

Like many studies on invariant manifolds of dynamical systems, our work is based on the standard Hadamard graph transform technique ([22]). However, with a general invariant manifold involved, several technical difficulties need to be resolved when we apply the Hadamard graph transform technique. The first one is to choose a function space to which the graph transform applies. In the equilibrium case, such a function space can be chosen as the space of Lipschitz functions from the center eigenspace to the hyperbolic eigenspaces. For the general case we consider, although an invariant splitting of the linearization along the invariant manifold is assumed, the 'center subspaces' associated to the invariant splitting do not form a manifold of desired dimension. Therefore, we need to construct an approximate center manifold which is tangent to the 'center subspaces'. This is done by introducing a new Riemannian structure on the tangent bundle of the invariant manifold and employing the exponential map. By defining a smooth bundle structure over the approximated center manifold in a neighborhood of the invariant manifold, a function space is then chosen as sections of the bundle. Another key step in applying the graph transform technique is to modify the original vector field near the invariant manifold to satisfy the so-called 'overflowing' property. Unlike the case of [19], the modified vector field in our case cannot be made to be $C^{1}$ close to the original one in general - which results in a large perturbation problem. Therefore, a careful choice and estimations on the modified vector field are crucial in our analysis.

Besides the Hadamard graph transform, another fruitful technique which has been frequently used in the classical center manifolds theory as well as other invariant manifolds (such as inertial and integral manifolds) theory is the LyapunovPerron method ([33, 38]). This method allows more detailed analysis on and near an invariant manifold especially when a natural coordinate system is available for 
a particular problem (e.g. 99, 20], [25, [30, 39], [56]). The Lyapunov-Perron method does not seem to directly apply to our problem. On one hand, to be able to work directly with integral equations as the Lyapunov-Perron method suggested, one more or less needs to construct a center manifold by obtaining its invariant foliations based on the invariant manifold. This is indeed the case of Chow and Lu (14]) in a nearly integrable system and the case of Chow and Yi ([15]) for flows with skew product structures. However, such foliations of a center manifold cannot be generally expected, for example, in many Hamiltonian systems. On the other hand, to apply the Lyapunov-Perron method in our current situation, a uniform separation rate associated to a linear invariant splitting should be generally assumed. But this would be a major restriction to many interesting applications.

This paper is organized as follows. We state our main results along with several examples in Section 2. In Section 3, we modify the original vector field near an invariant manifold of the flow following the constructions of an approximated centerunstable manifold and a local bundle structure. Section 4 is a rather technical section which is devoted to the estimations of the modified flow. To avoid reading too much technical details, one may skip most materials in this section and only take Proposition 1 for granted. Our main theorem is proved in Section 5.

\section{Main Results AND EXAMPles}

2.1. Statement of main theorem. Consider the following ordinary differential equation

$$
z^{\prime}=f(z)
$$

where $z \in \mathbb{R}^{n}, f \in C^{r}(r \geq 3)$.

Let $Y$ be a smooth invariant manifold of (11). For simplicity, we denote the induced flow on $Y$ by $y \cdot t(y \in Y, t \in \mathbb{R})$. Consider the linearization of (1) along $Y$ :

$$
z^{\prime}=A(y \cdot t) z
$$

where $A(y)=J f(y)$ is the Jacobian of $f$ at $y \in Y$. We let $\Phi(y, t)$ be the principal matrix of (2), i.e., the fundamental matrix solution of (2) with $\Phi(y, 0)=I$ - the identity matrix.

We make the following hypotheses.

(H1.) System (2) admits a continuous invariant splitting of the tangent spaces $T_{y} \mathbb{R}^{n}(y \in Y)$, that is,

$$
T_{y} \mathbb{R}^{n}=V_{s}(y) \oplus V_{c}(y) \oplus V_{u}(y),
$$

where $T_{y} Y \subset V_{c}(y), V_{i}(y)$ varies continuously in $y \in Y$ and $\Phi(y, t) V_{i}(y)=V_{i}(y \cdot t)$, $i=s, c, u$, for all $t \in \mathbb{R}$ and $y \in Y$.

(H2.) With respect to a fixed Riemannian structure $\mathcal{R}$ on the tangent bundle $T_{Y} \mathbb{R}^{n}$, there exist constants $\alpha, \beta$ and integer $d$ satisfying $0<d \leq r, \alpha<0$, and $0 \leq \beta<\frac{1}{d}$ such that

$$
\begin{aligned}
\left\|\left.\Phi(y, t)\right|_{V_{s}(y)}\right\| & \leq e^{\alpha t}, \quad t \geq 0 \\
\left\|\left.\Phi(y, t)\right|_{V_{u}(y)}\right\| & \leq e^{-\alpha t}, \quad t \leq 0 ; \\
m\left(\left.\Phi(y, t)\right|_{V_{c}(y)}\right) & \geq\left\|\left.\Phi(y, t)\right|_{V_{s}(y)}\right\|^{\beta}, \quad t \geq 0 \\
m\left(\left.\Phi(y, t)\right|_{V_{c}(y)}\right) & \geq\left\|\left.\Phi(y, t)\right|_{V_{u}(y)}\right\|^{\beta}, \quad t \leq 0
\end{aligned}
$$


for all $y \in Y$, where, for a linear operator $L,\|L\|$ denotes the operator norm of $L$ with respect to $\mathcal{R}$ and $m(L)=\min \{\|L z\|:\|z\|=1\}$.

For $i=s, c, u$, and $y \in Y$, if we denote $P_{i}(y): T_{y} \mathbb{R}^{n} \rightarrow V_{i}(y)$ as the projections associated to the invariant splitting, then $\left.\Phi(y, t)\right|_{V_{i}(y)}=\Phi(y, t) P_{i}(y)$. For simplicity, sometimes we shall also denote $\left.\Phi(y, t)\right|_{V_{i}(y)}$ as $\Phi_{i}(y, t)$ for $i=s, c, u$ respectively.

Remark 1. Since $\Phi(y, t) \Phi^{-1}(y, s)=\Phi(y \cdot s, t-s), P_{i}(y \cdot t) \Phi(y, t)=\Phi(y, t) P_{i}(y)$, one has $\Phi(y, t) P_{i}(y) \Phi^{-1}(y, s)=\Phi(y \cdot s, t-s) P_{i}(y \cdot s)(i=s, c, u)$ for all $t, s$ and $y \in Y$. It follows that the first two inequalities in (H2.) are equivalent to

$$
\begin{aligned}
& \left\|\Phi(y, t) P_{s}(y) \Phi^{-1}(y, s)\right\| \leq e^{\alpha(t-s)}, \quad t \geq s, \quad y \in Y \\
& \left\|\Phi(y, t) P_{s}(y) \Phi^{-1}(y, s)\right\| \leq e^{-\alpha(t-s)}, \quad t \leq s, \quad y \in Y
\end{aligned}
$$

respectively.

Definition 1. (a) A submanifold $M$ of $\mathbb{R}^{n}$ with boundary $\partial M$ is called locally invariant under (1), if, for any point $p \in M \backslash \partial M$, there exists an $\epsilon>0$ such that $z(t, p) \in M$ for $t \in(-\epsilon, \epsilon)$, where $z(t, p)$ is the solution of (11) with $z(0, p)=p$.

(b) A locally invariant $C^{k}(k \leq r)$ manifold $M$ of a $C^{r}$ vector field $f$ is called $C^{k}$ persistent if there exists a neighborhood $\mathcal{U}(f)$ of $f$ in the space of $C^{k}$ vector fields with the $C^{k}$ topology, such that for each $g \in \mathcal{U}(f)$, there exists a locally invariant $C^{k}$ manifold $M(g)$ (not necessarily unique) of $g$ which is $C^{k}$ close to $M$ with respect to the Hausdorff metric.

Our main theorem is stated as follows.

Theorem 1. Suppose $Y$ is a connected, invariant and closed $C^{r}$ manifold of (1) for which $(\mathbf{H 1})$ and $\mathbf{( H 2 )}$ are satisfied. Then there exists a manifold $M_{c}(Y)$ with the following properties.

(i) $M_{c}(Y)$ is $C^{d}$ and locally invariant;

(ii) $T_{y} M_{c}(Y)=V_{c}(y)$ for all $y \in Y$;

(iii) $M_{c}(Y)$ is $C^{d}$ persistent;

(iv) $M_{c}(Y)$ contains all locally bounded solutions, that is, there exists a neighborhood $N(Y)$ of $Y$ such that $M_{c}(Y)$ contains all solutions lying entirely in $N(Y)$.

The property (iv) above particularly implies that $M_{c}(Y)$ contains $Y$.

We refer to a manifold $M_{c}(Y)$ as a center manifold of $Y$ if all properties (i)-(iv) above are satisfied. By (ii) and (iii) above, if the invariant splitting in (H1) is 'optimal', then $M_{c}(Y)$ more or less gives a normally hyperbolic invariant manifold containing $Y$ with the least dimension (comparing (iii) with [6], [34]).

We shall leave the proof of this theorem to Section 5.

2.2. Spectral conditions. A natural (but not necessarily optimal) linear invariant splitting satisfying (H1) and (H2) above can be constructed by applying the well known Sacker-Sell ([4]]) spectral theory (see also [46]).

Consider for each $\lambda \in \mathbb{R}$ the skew-product flow on $T_{Y} \mathbb{R}^{n}=\mathbb{R}^{n} \times Y$ :

$$
\pi_{\lambda}(z, y, t)=\left(\Phi_{\lambda}(y, t) z, y \cdot t\right)
$$

where $\Phi_{\lambda}(y, t)=e^{-\lambda t} \Phi(y, t)$ is the principal matrix of

$$
z^{\prime}=(A(y \cdot t)-\lambda) z .
$$


The flow $\pi_{\lambda}$ is said to admit an exponential dichotomy (ED) over $Y$, if there exist a continuous family of projections $P(y): T_{y} \mathbb{R}^{n} \rightarrow T_{y} \mathbb{R}^{n}(y \in Y)$ and constants $K>0, \alpha<0$ such that

$$
\begin{aligned}
\left|\Phi_{\lambda}(y, t) P(y) \Phi_{\lambda}^{-1}(y, s)\right| & \leq K e^{\alpha(t-s)}, \quad t \geq s \\
\left|\Phi_{\lambda}(y, t)[I-P(y)] \Phi_{\lambda}^{-1}(y, s)\right| & \leq K e^{-\alpha(t-s)}, \quad t \leq s,
\end{aligned}
$$

where $|\cdot|$ denotes the operator norm with respect to the Euclidean metric on $\mathbb{R}^{n}$.

The set

$$
\Sigma(Y)=\left\{\lambda \in \mathbb{R}: \pi_{\lambda} \text { admits no ED over } Y\right\}
$$

is called the $S$-S spectrum of (2).

Let

$$
\begin{aligned}
& \mathcal{S}_{\lambda}(Y)=\left\{(z, y):\left|\Phi_{\lambda}(y, t) z\right| \rightarrow 0 \text { as } t \rightarrow+\infty\right\}, \\
& \mathcal{U}_{\lambda}(Y)=\left\{(z, y):\left|\Phi_{\lambda}(y, t) z\right| \rightarrow 0 \text { as } t \rightarrow-\infty\right\} .
\end{aligned}
$$

The following was shown in 43 .

1) $\Sigma(Y)$ is a union of $k$ compact intervals, that is,

$$
\Sigma(Y)=\left[a_{1}, b_{1}\right] \cup \cdots \cup\left[a_{k}, b_{k}\right],
$$

where $k \leq n$ and $a_{1} \leq b_{1}<a_{2} \leq b_{2}<\cdots<a_{k} \leq b_{k}$.

2) If $\lambda_{0}, \lambda_{1}, \cdots, \lambda_{k}$ are chosen so that

$$
\lambda_{0}<a_{1} \leq b_{1}<\lambda_{1}<\cdots \leq b_{k}<\lambda_{k},
$$

then for $1 \leq i \leq k$

$$
V_{i}=V_{i}(Y)=\mathcal{S}_{\lambda_{i}}(Y) \cap \mathcal{U}_{\lambda_{i-1}}(Y) \neq\{0\} \times Y
$$

is an invariant subbundle (called the spectral subbundle associated to the spectral interval $\left.\left[a_{i}, b_{i}\right]\right)$ of $T_{Y} \mathbb{R}^{n}$. Moreover, $V_{i}(Y) \cap V_{j}(Y)=\{0\} \times Y$ for $i \neq j$ and

$$
T_{Y} \mathbb{R}^{n}=V_{1}(Y) \oplus \cdots \oplus V_{k}(Y) .
$$

In terms of fibers $V_{i}(y)(y \in Y)$ of $V_{i}$, the above is equivalent to

$$
T_{y} \mathbb{R}^{n}=V_{1}(y) \oplus \cdots \oplus V_{k}(y)
$$

for all $y \in Y$, where $\operatorname{dim} V_{i}(y)=n_{i}\left(y \in Y, n_{i} \geq 1\right.$ and $\left.n_{1}+n_{2}+\cdots+n_{k}=n\right)$.

Now consider, for $i \leq j$, a union of spectral intervals of form $\Sigma_{i, j}=\bigcup_{p=i}^{j}\left[a_{p}, b_{p}\right]$ and denote the corresponding spectral subbundle by $V_{i, j}=V_{i} \oplus \cdots \oplus V_{j}$. Let

$$
i_{0}=\max \left\{i: T Y \subset V_{i, k}\right\}, \quad j_{0}=\min \left\{j: T Y \subset V_{1, j}\right\},
$$

where $T Y$ is the tangent bundle of $Y$, that is, $V_{i_{0}, j_{0}}$ is the smallest spectral subbundle which contains $T Y$. Clearly, $i_{0}$ and $j_{0}$ are uniquely defined. We refer to $\Sigma_{c}=\Sigma_{i_{0}, j_{0}}, V_{c}=V_{i_{0}, j_{0}}$ as the generalized center spectrum and the generalized center subbundle of $Y$, respectively. Note that, since $Y$ is compact and invariant, $0 \in \Sigma_{c}$, and $\Sigma_{c}$ may contain more than one spectral intervals (see Example 2.2.). To unify the notation, we let $a_{j_{0}+1}=+\infty$ if $i_{0}=k$ and let $b_{i_{0}-1}=-\infty$ if $j_{0}=1$.

Corollary 1. Let $Y$ be as in Theorem 1 and let $\Sigma_{c}=\Sigma_{i_{0}, j_{0}}$ be the generalized center spectrum of $Y$ for which the following spectrum gap conditions are satisfied: there is a positive integer $d \leq r$ such that

$$
-a_{i_{0}} d<-b_{i_{0}-1}, \quad b_{j_{0}} d<a_{j_{0}+1} .
$$


Then there exists a manifold $M_{c}(Y)$ which satisfies all properties (i)-(iv) stated in Theorem 1 .

Proof. Without loss of generality, we assume that $a_{j_{0}+1}$ and $b_{i_{0}-1}$ are finite.

Consider (2) and denote

$$
V_{s}(y)=V_{1, i_{0}-1}(y), \quad V_{u}(y)=V_{j_{0}+1, k}(y) \quad(y \in Y) .
$$

Let

$$
P_{i}(y): T_{y} \mathbb{R}^{n} \rightarrow V_{i}(y), \quad y \in Y, i=s, c, u
$$

be the associated projections. Then

$$
T_{y} \mathbb{R}^{n}=V_{s}(y) \oplus V_{c}(y) \oplus V_{u}(y), \quad y \in Y,
$$

is a continuous invariant splitting of (2).

We now define an equivalent metric on $T_{Y} \mathbb{R}^{n}$ following the ideas of [26, [28, [42]. Fix a $0<\lambda<\min \left\{\left(a_{i_{0}}-b_{i_{0}-1}\right) / 2,\left(a_{j_{0}+1}-b_{j_{0}}\right) / 2\right\}$. For $y \in Y$ and $z_{1}, z_{2} \in T_{y} \mathbb{R}^{n}$, we let

$$
\begin{aligned}
\left\langle z_{1}, z_{2}\right\rangle_{y}= & \int_{-\infty}^{0} e^{-2\left(a_{j_{0}+1}-\lambda\right) s}\left\langle\Phi(y, s) P_{u}(y) z_{1}, \Phi(y, s) P_{u}(y) z_{2}\right\rangle d s \\
& +\int_{-\infty}^{0} e^{-2\left(a_{i_{0}}-\lambda\right) s}\left\langle\Phi(y, s) P_{c}(y) z_{1}, \Phi(y, s) P_{c}(y) z_{2}\right\rangle d s \\
& +\int_{0}^{+\infty} e^{-2\left(b_{j_{0}}+\lambda\right) s}\left\langle\Phi(y, s) P_{c}(y) z_{1}, \Phi(y, s) P_{c}(y) z_{2}\right\rangle d s \\
& +\int_{0}^{+\infty} e^{-2\left(b_{i_{0}-1}+\lambda\right) s}\left\langle\Phi(y, s) P_{s}(y) z_{1}, \Phi(y, s) P_{s}(y) z_{2}\right\rangle d s .
\end{aligned}
$$

Since

$$
\begin{aligned}
& \left|\Phi(y, t) P_{s}(y) \Phi^{-1}(y, s)\right| \leq K e^{\left(b_{i_{0}-1}+\lambda / 2\right)(t-s)}, \quad t \geq s \\
& \left|\Phi(y, t) P_{c}(y) \Phi^{-1}(y, s)\right| \leq K e^{\left(b_{j_{0}}+\lambda / 2\right)(t-s)}, \quad t \geq s \\
& \left|\Phi(y, t) P_{c}(y) \Phi^{-1}(y, s)\right| \leq K e^{\left(a_{i_{0}}-\lambda / 2\right)(t-s)}, \quad t \leq s \\
& \left|\Phi(y, t) P_{u}(y) \Phi^{-1}(y, s)\right| \leq K e^{\left(a_{j_{0}+1}-\lambda / 2\right)(t-s)}, \quad t \leq s
\end{aligned}
$$

for some $K>0$, the above integral converges and clearly defines an inner product $\langle\cdot, \cdot\rangle_{y}$ on $T_{y} \mathbb{R}^{n}(y \in Y)$. We denote $\|\cdot\|_{y}$ and $\|\cdot\|$, respectively, as the induced norm on $T_{y} \mathbb{R}^{n}(y \in Y)$ and its associated operator norm, respectively. To show the uniform equivalence between $\|\cdot\|_{y}$ and the Euclidean norm $|\cdot|$ on $T_{y} \mathbb{R}^{n}(y \in Y)$, 
on one hand, we have

$$
\begin{aligned}
& \left\|z_{0}\right\|_{y}^{2}=\int_{-\infty}^{0} e^{-2\left(a_{j_{0}+1}-\lambda\right) s}\left|\Phi(y, s) P_{u}(y) z_{0}\right|^{2} d s \\
& +\int_{-\infty}^{0} e^{-2\left(a_{i_{0}}-\lambda\right) s}\left|\Phi(y, s) P_{c}(y) z_{0}\right|^{2} d s \\
& +\int_{0}^{+\infty} e^{-2\left(b_{j_{0}}+\lambda\right) s}\left|\Phi(y, s) P_{c}(y) z_{0}\right|^{2} d s \\
& +\int_{0}^{+\infty} e^{-2\left(b_{i_{0}-1}+\lambda\right) s}\left|\Phi(y, s) P_{s}(y) z_{0}\right|^{2} d s \\
& \leq \int_{-\infty}^{0} e^{-2\left(a_{j_{0}+1}-\lambda\right) s} K^{2} e^{2\left(a_{j_{0}+1}-\lambda / 2\right) s}\left|z_{0}\right|^{2} d s \\
& +\int_{-\infty}^{0} e^{-2\left(a_{i_{0}}-\lambda\right) s} K^{2} e^{2\left(a_{i_{0}}-\lambda / 2\right) s}\left|z_{0}\right|^{2} d s \\
& +\int_{0}^{+\infty} e^{-2\left(b_{j_{0}}+\lambda\right) s} K^{2} e^{2\left(b_{j_{0}}+\lambda / 2\right) s}\left|z_{0}\right|^{2} d s \\
& +\int_{0}^{+\infty} e^{-2\left(b_{i_{0}-1}+\lambda\right) s} K^{2} e^{2\left(b_{i_{0}-1}+\lambda / 2\right) s}\left|z_{0}\right|^{2} d s \\
& =\left(2 K^{2} \int_{-\infty}^{0} e^{\lambda s} d s+2 K^{2} \int_{0}^{+\infty} e^{-\lambda s} d s\right)\left|z_{0}\right|^{2} \\
& =\frac{4 K^{2}}{\lambda}\left|z_{0}\right|^{2} \text {. }
\end{aligned}
$$

On the other hand, since $Y$ is compact and $\Phi(y, 0)=I$, there exists a $\delta>0$ such that $|\Phi(y, s)| \geq 1 / 2$ as $|s| \leq \delta$. It follows that

$$
\begin{aligned}
\left\|z_{0}\right\|_{y}^{2} \geq & \frac{1}{4} \int_{-\delta}^{0} e^{-2\left(a_{j_{0}+1}-\lambda\right) s}\left|P_{u}(y) z_{0}\right|^{2} d s+\frac{1}{4} \int_{-\delta}^{0} e^{-2\left(a_{i_{0}}-\lambda\right) s}\left|P_{c}(y) z_{0}\right|^{2} d s \\
& \frac{1}{4}+\int_{0}^{\delta} e^{-2\left(b_{j_{0}}+\lambda\right) s}\left|P_{c}(y) z_{0}\right|^{2} d s+\frac{1}{4} \int_{0}^{\delta} e^{-2\left(b_{i_{0}-1}+\lambda\right) s}\left|P_{s}(y) z_{0}\right|^{2} d s \\
\geq & c_{2}^{-1}\left(\left|P_{u}(y) z_{0}\right|^{2}+\left|P_{c}(y) z_{0}\right|^{2}+\left|P_{s}(y) z_{0}\right|^{2}\right) \geq c_{2}^{-1}\left|z_{0}\right|^{2}
\end{aligned}
$$

for some $c_{2}>0$ which is independent of $y \in Y$. This verifies the equivalence of the two metrics.

Since

$$
\begin{aligned}
\left\|\Phi(y, t) P_{s}(y) z_{0}\right\|_{y \cdot t}^{2} & =\int_{0}^{+\infty} e^{-2\left(b_{i_{0}-1}+\lambda\right) s}\left|\Phi(y \cdot t, s) \Phi(y, t) P_{s}(y) z_{0}\right|^{2} d s \\
& =\int_{0}^{+\infty} e^{-2\left(b_{i_{0}}-1+\lambda\right) s}\left|\Phi(y, s+t) P_{s}(y) z_{0}\right|^{2} d s \\
& =\int_{t}^{+\infty} e^{-2\left(b_{i_{0}}-1+\lambda\right)(s-t)}\left|\Phi(y, s) P_{s}(y) z_{0}\right|^{2} d s \\
& \leq e^{2\left(b_{i_{0}-1}+\lambda\right) t} \int_{0}^{\infty} e^{-2\left(b_{i_{0}}-1+\lambda\right) s}\left|\Phi(y, s) P_{s}(y) z_{0}\right|^{2} d s \\
& =e^{2\left(b_{i_{0}-1}+\lambda\right) t}\left|z_{0}\right|_{y}^{2},
\end{aligned}
$$




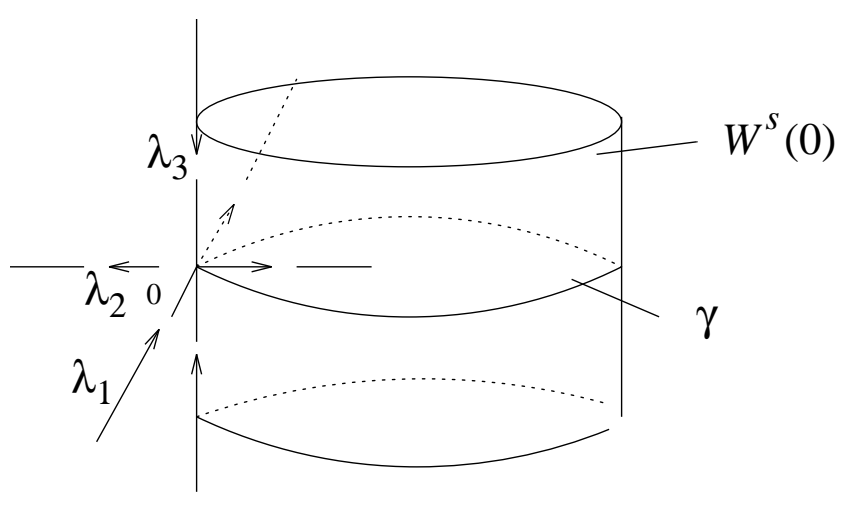

Figure 1. Stable set $W^{s}(0)$ of 0 along $\gamma$

for any $t \geq 0$, we have

$$
\left\|\Phi(y, t) P_{s}(y) \Phi^{-1}(y, s)\right\| \leq e^{\left(b_{i_{0}-1}+\lambda\right)(t-s)}
$$

for $t \geq s$. Similarly,

$$
\begin{gathered}
\left\|\Phi(y, t) P_{c}(y) \Phi^{-1}(y, s)\right\| \leq e^{\left(b_{j_{0}}+\lambda\right)(t-s)}, \quad t \geq s \\
\left\|\Phi(y, t) P_{c}(y) \Phi^{-1}(y, s)\right\| \leq e^{\left(a_{i_{0}}-\lambda\right)(t-s)}, \quad t \leq s \\
\left\|\Phi(y, t) P_{u}(y) \Phi^{-1}(y, s)\right\| \leq e^{\left(a_{j_{0}+1}-\lambda\right)(t-s)}, \quad t \leq s .
\end{gathered}
$$

Now let $\alpha=\max \left\{b_{i_{0}-1}+\lambda, \lambda-a_{j_{0}+1}\right\}$ and choose $\beta$ such that $\frac{a_{i_{0}}-\lambda}{b_{i_{0}}-1+\lambda} \leq \beta<$ $\min \left\{\frac{b_{j_{0}}}{a_{j_{0}+1}}, \frac{a_{i_{0}}}{b_{i_{0}-1}}\right\}$. It is easy to see from the above inequalities that (H2) is satisfied with such $\alpha$ and $\beta$.

2.3. Examples. We now give some examples to illustrate certain fundamental aspects of our results. The first is an example to which Corollary 1 is applicable.

Example 2.1. Consider a flow

$$
z^{\prime}=f(z), \quad z \in \mathbb{R}^{3}, \quad f \in C^{r}
$$

where $z=0$ is a saddle-node equilibrium with eigenvalues $\lambda_{i}, i=1,2,3$, satisfying $\lambda_{3}<\lambda_{1}=0<\lambda_{2}$. Assume that $\gamma(t)$ is a homoclinic orbit to $z=0$ which approaches $z=0$ along the eigendirection of $\lambda_{1}$ as $t \rightarrow \pm \infty$ (see Fig. 1).

Let $Y=\{\gamma(t) ; t \in \mathbb{R}\} \cup\{0\}$. We further assume that, in a neighborhood of $\gamma(t)$, the closure of the stable set $W^{s}(0)$ of $z=0$ is a manifold (i.e. a cylinder or a Möbius band). By the Lambda Lemma ([1], [49]), $W^{u}(0)$ is also a manifold of the same topology type as $W^{s}(0)$, and, on the stable set of 0 , there exists a unique locally invariant stable foliation of $W^{s}(0)$ with fibers transversal to $Y$ (see [1], [2]). By the Lambda Lemma again, the foliation can be extended to the entire $W^{s}(0)$ by using the backward flow. Similarly, there exists an invariant unstable foliation on $W^{u}(0)$. Therefore, for $y \in Y$, the tangent lines to the fibers at $y$ of the stable and unstable foliations together with $T_{y} Y$ form an invariant splitting of $T_{y} \mathbb{R}^{3}$ of the linearization along $Y$. It can be easily verified that the S-S spectrum of the linearization along $Y$ is $\Sigma=\left\{\lambda_{1}, \lambda_{2}, \lambda_{3}\right\}$ and the spectral subbundle corresponding to $\left\{\lambda_{1}=0\right\}$ is $T Y$. 
Thus, conditions in Corollary 1 are satisfied with $V_{c}(y)=T_{y} Y(y \in Y)$. We then conclude that the flow admits a one-dimensional $C^{r}$ center manifold of $Y$, that is, $Y$ itself.

Next, we adopt an example from [43] to indicate the necessity of choosing the generalized center spectrum when applying Corollary 1 .

Example 2.2. Let $T^{2}=\mathbb{R}^{2} / \mathbb{Z}^{2}$ be the two-dimensional torus. As usual, we coordinate $x \in T^{2}$ by $x=\left(x_{1}, x_{2}\right) \in \mathbb{R}^{2}(\bmod 1)$. Let $\bar{x}=A x$ be the Anosov diffeomorphism on $T^{2}$, where

$$
A=\left(\begin{array}{ll}
1 & 1 \\
1 & 2
\end{array}\right)
$$

The eigenvalues of $A$ are $\sigma_{ \pm}=(3 \pm \sqrt{5}) / 2$.

Let $z^{\prime}=F(z)$ denote the Anosov flow on a 3-manifold $Y$ generated by the standard suspension of the Anosov diffeomorphism. Recall that $Y$ can be identified as the collection of all $z=(x, s)$ where $x=\left(x_{1}, x_{2}\right) \in \mathbb{R}^{2}, s \in \mathbb{R}$, and $(x, s)=(\hat{x}, \hat{s})$ if and only if $\hat{x}=A^{n} x(\bmod 1)$ and $\hat{s}=s+n$ for some integer $n$. Since the Anosov diffeomorphism on $T^{2}$ is a Poincaré map of the Anosov flow, the S-S spectrum of the linearization of the Anosov flow along $Y$ is simply $\Sigma(Y)=\{\lambda,-\lambda, 0\}$ where $\lambda=\ln \sigma_{+}$.

We now consider the following flow on the four-dimensional manifold $W=Y \times$ $S^{1}$ :

$$
\begin{aligned}
& z^{\prime}=F(z)+h(z, \theta), \\
& \theta^{\prime}=\alpha \sin \theta+g(z, \theta),
\end{aligned}
$$

where $(z, \theta) \in W, \alpha$ is a parameter, $h$ and $g$ are smooth functions satisfying $h(z, 0)=0$ and $|g(z, \theta)|=O\left(|\theta|^{2}\right)$. Clearly, $Y$ is an invariant manifold of $W$ given by $\theta=0$ and the S-S spectrum of the linearization of this flow along $Y$ is $\{\alpha, \lambda,-\lambda, 0\}$. Moreover, it follows from the suspension procedure that the spectral subbundle corresponding to 0 is of dimension one. But $Y$ is of dimension three, which implies that its center manifold is at least three dimensional. Therefore, considering the spectral interval $\{0\}$ alone is not sufficient to generate a desired center manifold of $Y$. In fact, if $|\alpha|>\lambda$, then $Y$ is normally hyperbolic and the center manifold of $Y$ is just $Y$ itself. If $|\alpha|<\lambda$, then $Y$ is not normally hyperbolic and a center manifold of $Y$ is of dimension four, that is, a neighborhood of $Y$ in $W$.

We now give an example in which Corollary 1 fails but Theorem 1 can be still applied to obtain an optimal center manifold for an invariant manifold.

Example 2.3. Consider a flow

$$
z^{\prime}=f(z), \quad z \in \mathbb{R}^{2}, \quad f \in C^{r},
$$

which has two heteroclinic orbits $\gamma_{1}(t)$ and $\gamma_{2}(t)$ connecting a saddle point $z_{1}=$ $(-1,0)$ to a stable point $z_{2}=(1,0)$ (see Fig. 2). Denote by $\alpha_{i}, \beta_{i}$ as the eigenvalues of $z_{i}(i=1,2)$ respectively, where $\alpha_{1}<0<\beta_{1}, \alpha_{2}<\beta_{2}<0$. For simplicity, we assume that, for $i=1,2$, the eigendirections corresponding to $\alpha_{i}$ and $\beta_{i}$ are $e_{1}=(1,0)$ and $e_{2}=(0,1)$ respectively.

With the above assumptions, the closure $Y$ of $\gamma_{1} \cup \gamma_{2}$ is clearly a one dimensional invariant manifold of the flow. We denote by $\Sigma_{c}, \Sigma$, respectively, as the generalized center and S-S spectrum, respectively, of the linearization of the flow along $Y$. Since $\gamma_{1}^{\prime}(t)$ is a solution of the linearization along $\gamma_{1}$ with $\beta_{1}$ and $\beta_{2}$ as the asymptotic 

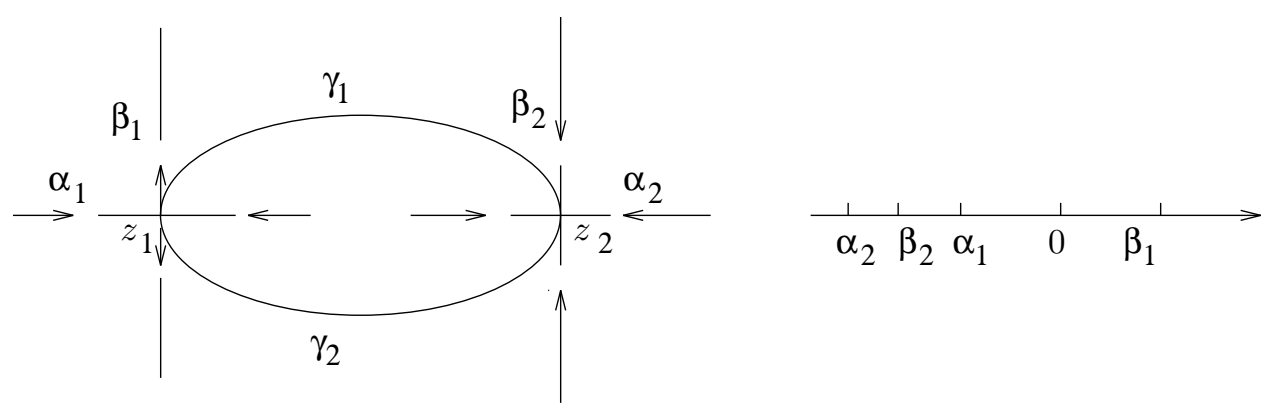

Figure 2. The invariant manifold $Y$ and the eigenvalues of the equilibria

rates, one has $\left[\beta_{2}, \beta_{1}\right] \subset \Sigma_{c}$. Hence, if $\alpha_{1} \geq \beta_{2}$, then $\Sigma_{c}=\Sigma=\left[\alpha_{2}, \beta_{1}\right]$ and the dimension of the generalized center spectral subbundle will be two. In this case, a center manifold of $Y$ obtained from Corollary 1 will be a neighborhood of $Y$ in $\mathbb{R}^{2}$.

However, we can apply Theorem 1 to show that, in any case, $Y$ is a center manifold of itself. Similar to the argument in Example 2.1 there exists a continuous invariant splitting of the linearization along $Y$, that is,

$$
T_{y} \mathbb{R}^{2}=V_{s}(y) \oplus V_{c}(y),
$$

where $V_{c}(y)=T_{y} Y$ and $V_{s}(y)$ is transversal to $T_{y} Y(y \in Y)$. Moreover, it is easy to see that there are positive constants $K(y)$ and $K\left(z_{1}\right)$ such that, for $y \in Y \backslash\left\{z_{1}\right\}$,

$$
\begin{aligned}
& \left|\Phi_{s}(y, t)\right| \leq K(y) e^{\alpha_{2} t}, \quad t \geq 0 \\
& \left|\Phi_{c}(y, t)\right| \geq K(y) e^{\beta_{2} t}, \quad t \geq 0
\end{aligned}
$$

and

$$
\begin{aligned}
& \left|\Phi_{s}\left(z_{1}, t\right)\right| \leq K\left(z_{1}\right) e^{\alpha_{1} t}, \quad t \geq 0 \\
& \left|\Phi_{c}\left(z_{1}, t\right)\right| \geq K\left(z_{1}\right) e^{\beta_{1} t}, \quad t \geq 0 ;
\end{aligned}
$$

where $\Phi_{i}(y, t)=\left.\Phi(y, t)\right|_{V_{i}(y)}(i=s, c)$ and $\Phi(y, t)$ denotes the principal matrix of the linearization along $Y$.

By the Uniformity Lemma in [18, there are constants $\alpha, \beta, K$ with $\alpha<0,0<$ $\frac{1}{\beta}<\frac{\alpha_{2}}{\beta_{2}}, K>0$ such that $\left|\Phi_{s}(y, t)\right| \leq K e^{\alpha t},\left|\Phi_{c}(y, t)\right| \geq K\left|\Phi_{s}(y, t)\right|^{\beta}$ for all $y \in Y$ and $t \geq 0$. Similar to the proof of Corollary 1, one can show that, for $y \in Y$ and $z_{1}, z_{2} \in T_{y} \mathbb{R}^{2}$

$$
\begin{aligned}
\left\langle z_{1}, z_{2}\right\rangle_{y}= & \int_{0}^{+\infty} e^{2(\alpha+\lambda) s}\left\langle\Phi_{s}(y, s) z_{1}, \Phi_{s}(y, s) z_{2}\right\rangle d s \\
& +\int_{0}^{\infty}\left\|\Phi_{s}(y, s)\right\|^{-2 \beta}\left\langle\Phi_{c}(y, s) z_{1}, \Phi_{c}(y, s) z_{2}\right\rangle d s
\end{aligned}
$$

defines an equivalent metric on $T_{Y} \mathbb{R}^{2}$, where $\lambda>0$ is a small constant. Moreover, if $\|\cdot\|$ denotes the operator norm with respect to the new metric, then

$$
\begin{aligned}
\left\|\Phi_{s}(y, t)\right\| & \leq e^{(\alpha+\lambda) t} ; \\
m\left(\Phi_{c}(y, t)\right) & \geq\left\|\Phi_{s}(y, t)\right\|^{\beta}
\end{aligned}
$$

for $t \geq 0$ (we note that, since $\operatorname{dim} V_{c}(y)=1, m\left(\Phi_{c}(y, t)\right)=\left\|\Phi_{c}(y, t)\right\|$ ). This verifies the condition (H2). 


\section{ModificAtion OF THE VECTOR FIELD}

We shall apply the graph transform induced by a time $T$-map $\phi^{T}$ of the flow to show the existence of a local center-unstable manifold. The existence of a local center-stable manifold can be obtained similarly after reversing time, and the intersection of the center-stable and the center-unstable manifolds in a neighborhood of $Y$ will give a desired local center manifold.

Our construction of a local center-unstable manifold will be based on the following crucial steps: 1) To find an approximate center-unstable manifold which will serve as a base space of graphs; 2) To define a bundle structure in a neighborhood of $Y$ in which graphs can be defined as sections; 3) To modify the original vector field locally so that the overflowing property is satisfied. These constructions are closely tied up together in a way that each later step depends heavily on the former ones.

3.1. An approximate center-unstable manifold. By an approximate centerunstable manifold of $Y$, we mean a smooth manifold which is tangent to $V_{c u}(y)=$ $V_{c}(y) \oplus V_{u}(y)$ at all points $y \in Y$.

Since $Y$ is smooth, such an approximate manifold can be obtained easily by using the exponential map (see [26] for example). Let $\mathcal{R}_{0}$ be a new Riemannian metric on $T_{Y} \mathbb{R}^{n}$ under which $V_{s}(y)$ is orthogonal to $V_{c u}(y)$ at each $y \in Y$. For a fixed neighborhood $N(Y)$ of $Y$, this metric can be extended smoothly to $T_{N(Y)} \mathbb{R}^{n}$ (we denote the extended metric again by $\left.\mathcal{R}_{0}\right)$. Let $V^{\prime}(Y)$ be the orthogonal complement of $T_{Y} Y$ in $V_{c u}(Y)$. For $\epsilon>0$ sufficiently small, we let $B(\epsilon)$ be the $\epsilon$-neighborhood of $Y$ in $T_{Y} \mathbb{R}^{n}$. Then $\hat{M}_{c u}(Y)=\exp \left(V^{\prime}(Y) \cap B(\epsilon)\right)$ defines a desired approximate center-unstable manifold since $T_{y} \hat{M}_{c u}(Y)=V_{c u}(y)$ for all $y \in Y$, where exp : $T_{N(Y)} \mathbb{R}^{n} \rightarrow \mathbb{R}^{n}$ is the exponential map with respect to $\mathcal{R}_{0}$.

3.2. A local bundle structure. We now construct a fiber bundle structure over $\hat{M}_{c u}(Y)$ in a neighborhood of $Y$.

By the tubular neighborhood theorem, there exists a neighborhood $N_{0}(Y)$ of $\hat{M}_{c u}(Y)$ in $N(Y)$ with the property that, for any $z \in N_{0}(Y)$, there exists a unique $p_{z} \in \hat{M}_{c u}(Y)$ with $\operatorname{dist}\left(z, \hat{M}_{c u}(Y)\right)=\operatorname{dist}\left(z, p_{z}\right)$. Define $\pi_{c u}: N_{0}(Y) \rightarrow \hat{M}_{c u}(Y)$ by $\pi_{c u}(z)=p_{z}, \pi_{s}: N_{0}(Y) \rightarrow N_{0}(Y)$ by $\pi_{s}(z)=z-\pi_{c u}(z):=q$, and $Q: \hat{M}_{c u}(Y) \rightarrow$ $Y$ by $Q(p)=y_{p}$, where $y_{p} \in Y$ is the unique point satisfying $\operatorname{dist}(p, Y)=\operatorname{dist}\left(p, y_{p}\right)$. By jiggling the bundle slightly if necessary, we can assume by Whitney's Embedding Theorem ([54 ) that the bundle and the maps defined above are $C^{r}$ (see also [18]). Denote the differentials of $\pi_{c u}, \pi_{s}$ at $z$ by $D \pi_{c u}(z)$ and $D \pi_{s}(z)$ respectively. We then have $D \pi_{c u}(y)=P_{c u}(y), D \pi_{s}(y)=P_{s}(y), y \in Y$.

The new Riemannian metric $\mathcal{R}_{0}$ under which $V_{c u}(y), V_{s}(y)$ are orthogonal will play an important role later in simplifying our analysis. Without loss of generality, we may assume that $\mathcal{R}_{0}$ agrees with the Euclidean metric in $\mathbb{R}^{n}$. This is because of Nash's Embedding Theorem ([36]), which says that any Riemannian $n$ manifold with $C^{r}$ metric, $r \geq 3$, has a $C^{r}$ isometric embedding in $n_{0}=3 / 2 n^{3}+$ $7 n^{2}+11 / 2 n$ dimensional Euclidean space. To be more precise, let $\left(N_{0}(Y), \mathcal{R}_{0}\right)$ be as above. By Nash's Embedding Theorem, it can be $C^{r}$ isometrically embedded in $\mathbb{R}^{n_{0}}$. By considering a tubular neighborhood $\mathcal{N}$ of $N_{0}(Y)$ in $\mathbb{R}^{n_{0}}$, we can extend our vector field to $\mathcal{N}$ in such a way that the extended flow on the fibers of $\mathcal{N}$ is unstable with sufficiently large rate. That is, the new flow in $\mathcal{N}$ is essentially the 


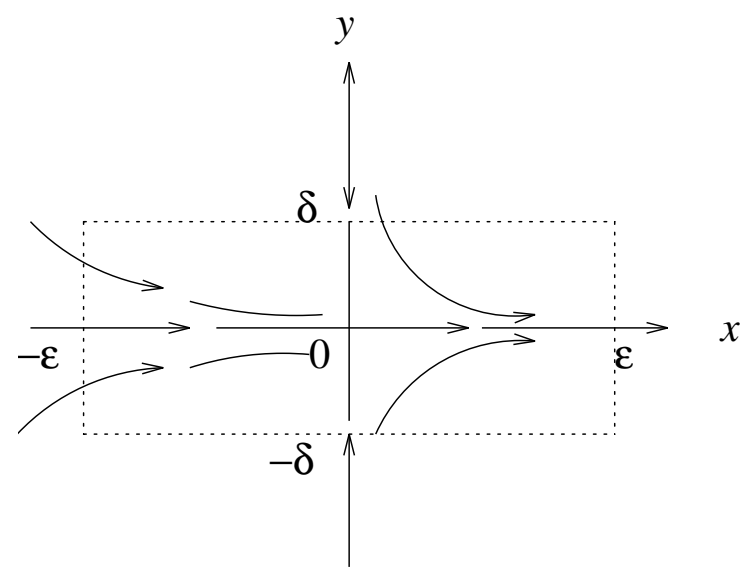

FIGURE 3. Lack of overflowing property

same as in $N_{0}(Y)$ with some extra unstable directions, which, of course, does not affect the existence of a center manifold.

In the sequel, we identify $\mathcal{R}_{0}$ with the Euclidean metric in $\mathbb{R}^{n}$ and denote $\langle\rangle,$, and $|\cdot|$ as the Euclidean metric, distance and norm, respectively.

\subsection{A modified vector field.}

Definition 2. Let $N \subset \mathbb{R}^{n}$ be a submanifold of dimension $n$ with smooth boundary $\partial N$, and $A \subset \partial N$. We say that a vector field $f$ satisfies the overflowing property with respect to $(N, A)$ if at each point $z \in A$ the vector field $f$ is either tangent to or points outward to $\partial N$.

The overflowing property is essential in performing a graph transform induced by a flow in a neighborhood of an invariant manifold (see [18, 19], [47] etc.). This is simply because the image of a graph under a graph transform should lie entirely in the neighborhood. For center manifolds problems, due to the existence of center directions in a vector field, the overflowing property cannot be generally expected. For example, even a simple vector field like

$$
\left\{\begin{array}{l}
x^{\prime}=x^{2} \\
y^{\prime}=-y
\end{array}\right.
$$

does not satisfy the overflowing property near $O=(0,0)$ for any choices of $\epsilon$ and $\delta$ (see Fig. 3). Specifically, on $x=-\epsilon$, the vector field points inward to the interior of the box $[-\epsilon, \epsilon] \times[-\delta, \delta]$.

Therefore, for the sake of performing a graph transform, we need to modify the vector field (11) so that the overflowing property will be satisfied within an appropriate neighborhood of $Y$. 
For $\epsilon_{0}>0$ small and $0<\epsilon \leq \epsilon_{0}$, we consider the following sets:

$$
\begin{aligned}
U_{\epsilon} & =\left\{z \in \hat{M}_{c u}(Y): d(z, Y) \leq \epsilon\right\}, \\
U\left(z_{0}\right) & =\left\{z \in N_{0}(Y): d\left(z, \hat{M}_{c u}(Y)\right)=d\left(z_{0}, \hat{M}_{c u}(Y)\right)\right\}, \quad z_{0} \in N_{0}(Y), \\
S\left(z_{0}\right) & =\left\{z \in N_{0}(Y): d\left(z, \hat{M}_{c u}(Y)\right)=d\left(z, z_{0}\right)\right\}, \quad z_{0} \in \hat{M}_{c u}(Y), \\
S_{\epsilon}\left(z_{0}\right) & =\left\{z \in S\left(z_{0}\right): d\left(z, z_{0}\right)<\epsilon\right\}, \quad z_{0} \in \hat{M}_{c u}(Y), \\
N_{\epsilon} & =\bigcup_{z_{0} \in U_{\epsilon}} S_{\epsilon_{0}}\left(z_{0}\right), \\
\partial^{*} N_{\epsilon} & =\bigcup_{z_{0} \in \partial U_{\epsilon}} S_{\epsilon_{0}}\left(z_{0}\right) .
\end{aligned}
$$

Note that $\partial^{*} N_{\epsilon}$ is the portion of $\partial N_{\epsilon}$ over $\partial U_{\epsilon}$, and $\operatorname{dim} \partial^{*} N_{\epsilon}=n-1$.

Our goal is to construct a modified vector field of (1) which satisfies the overflowing property with respect to $\left(N_{\epsilon_{0}}, \partial^{*} N_{\epsilon_{0}}\right)$. To do so, we let $\chi_{i}: \mathbb{R} \rightarrow \mathbb{R}, i=1,2$, be cut-off functions satisfying the following properties:

$$
\chi_{1}(x)= \begin{cases}1, & x \geq \epsilon_{0} / 2 \\ 0, & x \leq 0\end{cases}
$$

with $\chi_{1}^{\prime}(x) \geq 0,\left|\chi_{1}^{\prime}(x)\right| \leq 4 \epsilon_{0}^{-1}$, and

$$
\chi_{2}(x)= \begin{cases}0, & x \leq-\epsilon_{0}^{2}, \\ 1, & x \geq 0,\end{cases}
$$

with $\chi_{2}^{\prime}(x) \geq 0,\left|\chi_{2}^{\prime}(x)\right| \leq 2 \epsilon_{0}^{-2}$.

For $z \in \partial^{*} N_{\epsilon}$, let $\left\{v_{j}(z)\right\}_{j=1}^{n}$ be an orthonormal basis of $T_{z} N_{\epsilon}$ such that $v(z)=$ $v_{1}(z), v_{2}(z), \ldots, v_{m_{0}}(z) \in T_{z} U(z)$, where $v(z)$ is the inward normal vector to $T_{z} \partial^{*} N_{\epsilon}$ and $v_{m_{0}+1}, \ldots, v_{n}(z) \in T_{z} S_{\epsilon_{0}}\left(\pi_{c u} z\right)$. Using the orthonormal basis, we can decompose $f(z)=D \pi_{s}(z) f(z)+D \pi_{c u}(z) f(z)$ as

$$
\begin{aligned}
& D \pi_{c u}(z) f(z)=\sum_{j=1}^{m_{0}} \xi_{j}(z) v_{j}(z), \\
& D \pi_{s}(z) f(z)=\sum_{j=m_{0}+1}^{n} \xi_{j}(z) v_{j}(z),
\end{aligned}
$$

where $\xi_{j}(z)$ 's are coordinates of $f(z)$ with respect to the basis. Note that $\xi(z)=$ $\xi_{1}(z)=\langle f(z), v(z)\rangle$ is independent of the choices of $v_{j}(z)$ for $j=2, \cdots, n$.

We now modify the vector field (1) to the following:

$$
z^{\prime}=\hat{f}(z)=f(z)-\left(1-\chi_{1}(\eta(z))\right) \chi_{2}(\xi(z)) \xi(z) v(z)
$$

where $\eta(z)=d\left(\pi_{c u} z, \partial U_{\epsilon_{0}}\right)$. This new vector field clearly coincides with (1) in $N_{\epsilon_{0} / 2}$. Moreover, this modified vector field satisfies the overflowing property with respect to $\left(N_{\epsilon_{0}}, \partial^{*} N_{\epsilon_{0}}\right)$. Indeed, if $z \in \partial^{*} N_{\epsilon_{0}}$, then $\chi_{1}(\eta(z))=0$, and therefore

$$
\begin{aligned}
\langle\hat{f}(z), v(z)\rangle & =\langle f(z), v(z)\rangle-\chi_{2}(\xi(z)) \xi(z)\langle v(z), v(z)\rangle \\
& =\left(1-\chi_{2}(\xi(z))\right) \xi(z) .
\end{aligned}
$$

Since $\chi_{2}(\xi(z))=1$ if $\xi(z) \geq 0$, and $\left(1-\chi_{2}(\xi(z))\right) \geq 0$ if $\xi(z)<0$, we see that $\langle\hat{f}(z), v(z)\rangle \leq 0$ for $z \in \partial^{*} N_{\epsilon_{0}}$, that is, the vector field (3) is either tangent to or points outward to $\partial^{*} N_{\epsilon_{0}}$. 
Remark 2. Unlike the equilibrium case, the modified vector field (3) is in general not a regular perturbation of the original one in the sense that, within $N_{\epsilon_{0}}$, it differs from the original vector field by an order of $\epsilon_{0}$ with respect to the $C^{0}$ but not $C^{1}$ norm. To give an example, let us consider

$$
\left\{\begin{array}{l}
x^{\prime}=\lambda_{1} x+g_{1}(x, y) \\
y^{\prime}=\lambda_{2} y+g_{2}(x, y)
\end{array}\right.
$$

where $\lambda_{2}<\lambda_{1}<0$ and $g_{1}, g_{2}$ are higher order terms of $x$ and $y$. It is known that, near $O=(0,0)$, there exists a locally invariant manifold $Y$ which is tangent to the $x$-axis at $O=(0,0)$. If the above vector field is to be modified similarly to (3), say in a small box $[-\epsilon, \epsilon] \times[-\delta, \delta]$ near $Y$, then the modified vector field would be

$$
\left\{\begin{array}{l}
x^{\prime}=\chi(x)\left(\lambda_{1} x+g_{1}(x, y)\right) \\
y^{\prime}=\lambda_{2} y+g_{2}(x, y)
\end{array}\right.
$$

where $\chi(x)=0$ for $|x| \geq \epsilon$. Now, the Jacobian of the new vector field is

$$
\left(\begin{array}{cc}
\chi^{\prime}(x)\left(\lambda_{1} x+g_{1}\right)+\left(\lambda+\partial_{x} g_{1}\right) \chi(x) & \chi(x) \partial_{y} g_{1} \\
\partial_{x} g_{2} & \lambda_{2}+\partial_{y} g_{2}
\end{array}\right),
$$

which, at $( \pm \epsilon, 0)$, reduces to

$$
\left(\begin{array}{cc}
0+\text { h.o.t } & 0+\text { h.o.t } \\
\partial_{x} g_{2} & \lambda_{2}+\partial_{y} g_{2}
\end{array}\right)
$$

But the Jacobian of the original vector field at $( \pm \epsilon, 0)$ is simply

$$
\left(\begin{array}{cc}
\lambda_{1}+\text { h.o.t } & \text { h.o.t } \\
\partial_{x} g_{2} & \lambda_{2}+\partial_{y} g_{2}
\end{array}\right)
$$

where h.o.t stands for higher order terms of $x$ and $y$. Therefore, Jacobians for the two vector fields are not close to each other, that is, the new vector field is not $C^{1}$ close to the original one.

Although non-regular perturbations seem to be an unavoidable problem in the modification of vector fields of this type, our modified vector field (3) was chosen to single out the non-regular factors. Roughly speaking, near $\partial^{*} N_{\epsilon_{0}}$, we have projected the vector field $f(z)$ to $T_{z} \partial^{*} N_{\epsilon_{0}}$ for $z \in N_{\epsilon_{0}}$ and left the component along $V_{s}(Y)$ and $Y$ unchanged. Therefore, the components of the new vector field along the stable directions still remain as a regular perturbation to the original ones, that is, these components and their associated derivatives differ from the original ones by an order of $\epsilon_{0}^{2}$, and order of $\epsilon_{0}$, respectively (see Proposition 10) and Lemma 4). Consequently, the lower bound of the decay rate along these non-regular directions is close to the one associated to the original vector field (see Proposition 1 b)). The non-regular perturbation will only arise in the modification of the center-unstable directions, which however results in a slowing down of the modified flow when entering $U_{\epsilon_{0}}$. As we shall see in Section 4, such a 'slowing down' will actually provide a positive effect to our analysis.

\section{ANALYSis ON THE MODIFIED VECTOR FIELD}

Recall that for $0<\epsilon \leq \epsilon_{0}, N_{\epsilon}$ admits a bundle structure $N_{\epsilon}=\bigcup_{z_{0} \in U_{\epsilon}} S_{\epsilon_{0}}\left(z_{0}\right)$ over the approximate center-unstable manifold $\hat{M}_{c u}(Y)$. Throughout rest of the paper, for $z=p+q \in N_{\epsilon}$, where $p \in \hat{M}_{c u}(Y)$ and $q \in S_{\epsilon_{0}}(p)$, we simply use the notation $z=(p, q)$. 
In this section, we shall prove the following proposition which gives the key estimates to the modified flow.

Proposition 1. Given $T>0, C>0$ and $\epsilon_{0}$ small. If $\left(p_{i}, q_{i}\right) \in N_{\epsilon_{0}}$ and $z_{i}(t)=$ $\left(p_{i}(t), q_{i}(t)\right)$ are solutions of (3) with $z_{i}(0)=\left(p_{i}, q_{i}\right)(i=1,2)$ and $\left|p_{2}-p_{1}\right|<C \epsilon_{0}$, then there exists a $C(T)>0$ such that, as long as $z_{i}(t) \in N_{\epsilon_{0}}$ for $t \in[0, T]$, the following holds.

(a) (stable contracting rate)

$$
\begin{aligned}
\left|q_{2}(T)-q_{1}(T)\right| \leq & \left(e^{C(T) \epsilon_{0}}|| \Phi_{s}(y, T)||+C(T) \epsilon_{0}\right)\left|q_{2}-q_{1}\right| \\
& +C(T) \epsilon_{0}\left|p_{2}-p_{1}\right|,
\end{aligned}
$$

where $y=Q\left(p_{1}\right)$.

In particular,

$$
\left\|\left.D_{z}\left(\pi_{s} \phi^{T}\right)\right|_{T S_{\epsilon_{0}}(z)}\right\| \leq e^{C(T) \epsilon_{0}}|| \Phi_{s}(y, T) \|+C(T) \epsilon_{0},
$$

and

$$
\left.|| D_{z}\left(\pi_{s} \phi^{T}\right)\right|_{T U(z)} \| \leq C(T) \epsilon_{0},
$$

where $y=Q \pi_{c u} z$.

(b) (center-unstable expending rate)

$$
\begin{aligned}
\left|p_{2}(T)-p_{1}(T)\right| \geq & \left(e^{C(T) \epsilon_{0}}|| \Phi_{s}(y, T)||^{\beta}-C(T) \epsilon_{0}\right)\left|p_{2}-p_{1}\right| \\
& -C(T) \epsilon_{0}\left|q_{2}-q_{1}\right|,
\end{aligned}
$$

where $y=Q\left(p_{1}\right)$.

In particular,

$$
\left\|\left.D_{z}\left(\pi_{c u} \phi^{T}\right)\right|_{T U(z)}\right\| \geq e^{C(T) \epsilon_{0}}|| \Phi_{s}(y, T) \|^{\beta}-C(T) \epsilon_{0},
$$

where $y=Q \pi_{c u} z$.

We first study some properties of $v(z), \eta(z)$ and $\xi(z)$.

Lemma 1. There is a $C>0$ depending only on $Y$ such that for any $z \in \partial^{*} N_{\epsilon_{0}}$ the following holds.

(i) $v^{\top}(z) J v(z)=0,\left|J v(z) v_{j}(z)\right| \leq C \epsilon_{0}$ for $j \leq k_{0}$ or $j>m_{0}$, $\left|v_{j}^{\top}(z) J v(z) v_{j}(z)-\epsilon_{0}^{-1}\right| \leq C$ for $k_{0}<j \leq m_{0}$

(ii) $|\nabla \eta(z)-v(z)| \leq C \epsilon_{0}$;

(iii) $\left|\xi_{j}(z)\right| \leq C \epsilon_{0}$ for $j=1$ and $j>k_{0}$;

(iv) $|\nabla \xi(z)| \leq C$.

Proof. We identify $T_{y_{0}} Y, V^{\prime}\left(y_{0}\right)$ and $V_{s}\left(y_{0}\right)$ with $R^{k_{0}}, \mathbb{R}^{m_{0}-k_{0}}$ and $\mathbb{R}^{n-m_{0}}$, respectively. Let

$$
\mathcal{G}: N\left(y_{0}\right) \subset \mathbb{R}^{n} \rightarrow \mathbb{R}^{k_{0}} \times \mathbb{R}^{m_{0}-k_{0}} \times \mathbb{R}^{n-m_{0}}
$$

be the inverse of $\exp _{y_{0}}: T_{y_{0}} \mathbb{R}^{n} \rightarrow \mathbb{R}^{n}$. Then $D \mathcal{G}$ is $\epsilon_{0}$ close to the identity and $D^{2} \mathcal{G}$ is bounded. Moreover,

$$
\begin{aligned}
& \mathcal{G}\left(y_{0}\right)=0, \mathcal{G}(z)=\left(0, \cdots, 0, \epsilon_{0}, 0, \cdots, 0\right), \\
& \mathcal{G}: N\left(y_{0}\right) \cap Y \rightarrow \mathbb{R}^{k_{0}} \times\{0\} \times\{0\}, \text { and } \\
& \mathcal{G}: N\left(y_{0}\right) \cap U_{\epsilon_{0}} \rightarrow \mathbb{R}^{k_{0}} \times \mathbb{R}^{m_{0}-k_{0}} \times\{0\} \\
& \mathcal{G}\left(\partial U_{\epsilon_{0}}\right)=\mathcal{C}_{\epsilon_{0}},
\end{aligned}
$$




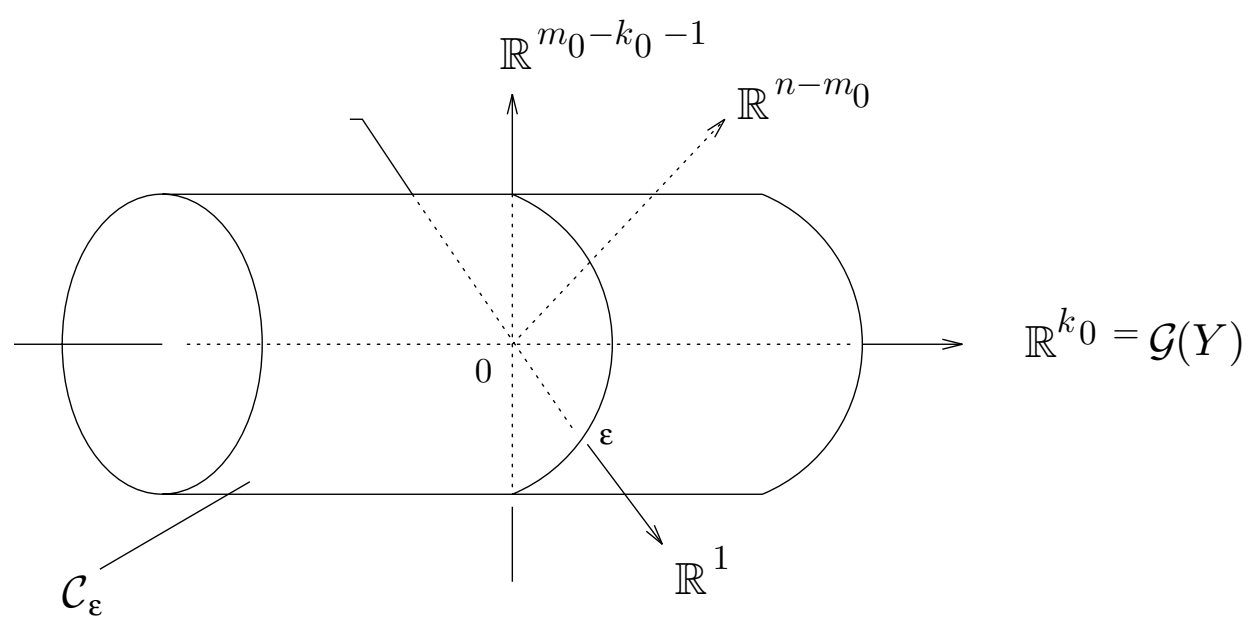

Figure 4. The image under $\mathcal{G}$

(see Fig. 4) where

$$
\mathcal{C}_{\epsilon}=\left\{x=\left(x_{1}, x_{2}, \cdots, x_{m_{0}}, 0, \cdots, 0\right): \sum_{j=k_{0}+1}^{m_{0}} x_{j}^{2}=\epsilon^{2}\right\} .
$$

To prove (i), we note that the inward normal vector at $x \in \mathcal{C}_{\epsilon}$ is

$$
n(x)=\frac{1}{|x|}\left(0, \cdots, 0,-x_{k_{0}+1}, \cdots,-x_{m_{0}}, 0, \cdots, 0\right) .
$$

Therefore,

$$
J n(x)=\frac{1}{|x|^{3}}\left(\begin{array}{ccc}
O_{k_{0} \times k_{0}} & O & O \\
O & D_{\left(m_{0}-k_{0}\right) \times\left(m_{0}-k_{0}\right)} & O \\
O & O & O_{\left(n-m_{0}\right) \times\left(n-m_{0}\right)}
\end{array}\right),
$$

where $D=\left(d_{i j}\right)$ with $d_{i i}=x_{i}^{2}-|x|^{2}$ and $d_{i j}=x_{i} x_{j}$ for $i \neq j, k_{0}+1 \leq i, j \leq m_{0}$. The matrix $D$ has zero as a simple eigenvalue with $n(x)$ as the corresponding eigenvector and has $-\frac{1}{|x|}$ as the other eigenvalues. If we assume, without loss of generality, that $x_{j}=0$ for $j \neq k_{0}+1$, then $d_{i j}=0$ for $i \neq j, d_{k_{0}+1, k_{0}+1}=0$ and $d_{i i}=-|x|^{-1}$ for $i \neq k_{0}+1$, that is, $D$ has a Jordan form with the desired eigenvalues. The property (i) easily follows.

Next, let $\bar{\eta}(x)=\epsilon_{0}-|x|$, for $x \in \mathcal{C}_{\epsilon}$, which is related to $\eta$ by $\mathcal{G}$. Since

$$
\nabla \bar{\eta}(x)=-\frac{1}{|x|}\left(0, \cdots, 0, x_{k_{0}+1}, \cdots, x_{m_{0}}, 0, \cdots, 0\right)^{\top}=n^{\top}(x),
$$

and $D \mathcal{G}$ is $\epsilon_{0}$ close to the identity, we have $|\nabla(\eta(z))-v(z)| \leq C \epsilon_{0}$. The property (ii) is proved.

The property (iii) clearly holds by the choice of $v_{j}$ 's.

It remains to prove (iv). Note that

$$
f(z)=\xi(z) v(z)+\sum_{j=2}^{n} \xi_{j}(z) v_{j}(z) .
$$


Then

$$
\begin{aligned}
J f(z)= & v(z)(\nabla \xi(z))^{\top}+\xi(z) J v(z) \\
& +\sum_{j=2}^{n}\left(v_{j}(z)\left(\nabla \xi_{j}(z)\right)^{\top}+\xi_{j}(z) J v_{j}(z)\right) .
\end{aligned}
$$

Multiplying the above equation by $v^{\top}(z)$ on the left, we obtain

$$
v^{\top}(z) A(z)=(\nabla \xi(z))^{\top}+\xi(z) v^{\top}(z) J v(z)+\sum_{j=2}^{n} \xi_{j}(z) v^{\top}(z) J v_{j}(z) .
$$

Since $\left\langle v_{i}(z), v_{j}(z)\right\rangle=\delta_{i j}$, we have

$$
v^{\top}(z) J v(z)=0 \text { and }\left(J v_{j}(z)\right)^{\top} v(z)=-(J v(z))^{\top} v_{j}(z) .
$$

Therefore,

$$
\begin{aligned}
\nabla \xi(z) & =A^{\top}(z) v(z)-\sum_{j=2}^{n} \xi_{j}(z)\left(J v_{j}(z)\right)^{\top} v(z) \\
& =A^{\top}(z) v(z)+\sum_{j=2}^{n} \xi_{j}(z)(J v(z))^{\top} v_{j}(z) .
\end{aligned}
$$

By (i) and (iii), $|\nabla \xi(z)| \leq C$.

The following generalized Gronwall's inequality will be frequently used later.

Lemma 2. If $\beta(t) \geq 0, \alpha(t)$ and $\phi(t)$ are continuous functions on $[a, b]$ and

$$
\phi(t) \leq \alpha(t)+\int_{a}^{t} \beta(s) \phi(s) d s, \quad a \leq t \leq b,
$$

holds, then

$$
\phi(t) \leq \alpha(t)+\int_{a}^{t} \beta(s) \alpha(s) e^{\int_{s}^{t} \beta(\mu) d \mu} d s, \quad a \leq t \leq b .
$$

If, in addition, $\alpha^{\prime}(t) \geq 0$, then $\phi(t) \leq \alpha(t) e^{\int_{a}^{t} \beta(s) d s}, a \leq t \leq b$.

Proof. See [24].

Lemma 3. Let $z_{1}(t)$ and $z_{2}(t)$ be trajectories of (11) and (3) respectively. For any $T$, if $z_{2}(t) \in N_{\epsilon_{0}}$ and $\lambda z_{2}(t)+(1-\lambda) z_{1}(t) \in N_{0}(Y)$, for all $|t| \leq T$ and $\lambda \in[0,1]$, then

$$
\left|z_{1}(t)-z_{2}(t)\right| \leq\left(\left|z_{2}(0)-z_{1}(0)\right|+C \epsilon_{0}|t|\right) e^{|D f||t|}
$$

for all $|t| \leq T$, where $|D f|=|D f|_{N_{0}(Y)}$ and $C$ is the constant defined in Lemma 1.

Proof. We only prove the case when $t \geq 0$. By (1) and (3),

$$
\begin{aligned}
\left(z_{1}(t)-z_{2}(t)\right)^{\prime}= & f\left(z_{1}(t)\right)-\hat{f}\left(z_{2}(t)\right) \\
= & f\left(z_{1}(t)\right)-f\left(z_{2}(t)\right) \\
& +\left(1-\chi_{1}\left(\eta\left(z_{2}(t)\right)\right)\right) \chi_{2}\left(\xi\left(z_{2}(t)\right)\right) \xi\left(z_{2}(t)\right) v\left(z_{2}(t)\right) .
\end{aligned}
$$


It follows that

$$
\begin{aligned}
& \left|z_{1}(t)-z_{2}(t)\right|-\left|z_{2}(0)-z_{1}(0)\right| \\
& \leq \int_{0}^{t}\left|f\left(z_{1}(s)\right)-f\left(z_{2}(s)\right)\right| d s+\int_{0}^{t}\left|\xi\left(z_{2}(s)\right) v\left(z_{2}(s)\right)\right| d s \\
& \leq|D f| \int_{0}^{t}\left|z_{1}(s)-z_{2}(s)\right| d s+\int_{0}^{t}\left|\xi\left(z_{2}(s)\right) v\left(z_{2}(s)\right)\right| d s .
\end{aligned}
$$

Since $\left|\xi\left(z_{2}(s)\right)\right| \leq C \epsilon_{0}$, Lemma 2 implies that

$$
\left|z_{1}(t)-z_{2}(t)\right| \leq\left(\left|z_{2}(0)-z_{1}(0)\right|+C \epsilon_{0} t\right) e^{|D f| t}, \quad 0 \leq t \leq T .
$$

This completes the proof.

Lemma 4. For a given $T>0$, there exists $C(T)>0$ such that if

$$
z(t)=(p(t), q(t)) \equiv\left(\pi_{c u} z(t), \pi_{s} z(t)\right)
$$

is the solution of (3) with $z(0)=\left(p_{0}, q_{0}\right) \in N_{\epsilon_{0}}$, then

$$
|q(t)| \leq e^{\alpha t}|z(0)-y|+C(T) \epsilon_{0}^{2}
$$

for $0 \leq t \leq T$, where $y=Q\left(p_{0}\right) \in Y$.

Proof. Below, we fix a $T>0$ and let $0 \leq t \leq T$.

Denote $F(z, y)=f(z)-f(y)-A(y)(z-y)$. Then

$$
\begin{aligned}
z^{\prime}(t)= & f(y \cdot t)+A(y \cdot t)(z(t)-y \cdot t)+F(z(t), y \cdot t) \\
& -\left(1-\chi_{1}(\eta(z))\right) \chi_{2}(\xi(z)) \xi(z(t)) v(z(t)) .
\end{aligned}
$$

The variation of constant formula yields that

$$
\begin{aligned}
z(t)-y \cdot t= & \Phi(y, t)(z(0)-y)+\int_{0}^{t} \Phi(y, t) \Phi^{-1}(y, s) F(z(s), y \cdot s) d s \\
& -\int_{0}^{t} \Phi(y, t) \Phi^{-1}(y, s)\left(1-\chi_{1}(\eta(z))\right) \chi_{2}(\xi(z)) \xi(z(s)) v(z(s)) d s .
\end{aligned}
$$

Therefore, by noting that $y \cdot t \in Y \subset \hat{M}_{c u}(Y)$, there exists a $C_{0}(T)>0$ such that

$$
\begin{aligned}
|q(t)| \leq & \left|\pi_{s} z(t)\right|=\left|\pi_{s} z(t)-\pi_{s}(y \cdot t)\right| \\
\leq & \left|D \pi_{s}(y \cdot t)(z(t)-y \cdot t)\right|+C_{0}(T)|z(t)-y \cdot t|^{2} \\
= & \left|P_{s}(y \cdot t)(z(t)-y \cdot t)\right|+C_{0}(T)|z(t)-y \cdot t|^{2} \\
\leq & \left|P_{s}(y \cdot t) \Phi(y, t)(z(0)-y)\right| \\
& +\left|\int_{0}^{t} \Phi(y, t) P_{s}(y) \Phi^{-1}(y, s) F(z(s), y \cdot s) d s\right| \\
& +\mid \int_{0}^{t} \Phi(y, t) P_{s}(y) \Phi^{-1}(y, s)\left(1-\chi_{1}(\eta(z(s)))\right) \chi_{2}(\xi(z(s))) \xi(z(s)) \\
& \cdot P_{s}(y \cdot s) v(z(s)) d s\left|+C_{0}(T)\right| z(t)-\left.y \cdot t\right|^{2} .
\end{aligned}
$$

By Lemma $3|z(s)-y \cdot s|=O\left(\epsilon_{0}\right)$ and hence $|F(z(s), y \cdot s)|=O\left(\epsilon_{0}^{2}\right)$. Since $v$ is tangent to $\hat{M}_{c u}(Y)$, we also have $\left|P_{s}(y \cdot s) v(z(s))\right|=O\left(\epsilon_{0}\right)$. The lemma then follows easily from the above inequality, (H2) and Remark 1, and Lemma 1 
Let $T>0$ and $z_{i}(t)=\left(p_{i}(t), q_{i}(t)\right)$ be solutions of (3) with $z_{i}(0)=\left(p_{i}, q_{i}\right) \in N_{\epsilon_{0}}$ $(i=1,2)$ such that $\left|p_{2}-p_{1}\right|=O\left(\epsilon_{0}^{2}\right)$. Denote $y=Q\left(p_{1}\right) \in Y$. By Lemma B, there is a $C(T)$ such that $\left|y \cdot t-z_{i}(t)\right| \leq C(T) \epsilon_{0}$ for $t \in[0, T]$.

Set $w(t)=z_{2}(t)-z_{1}(t)$. Since, by (3),

$$
\begin{aligned}
\left(z_{i}(t)-y \cdot t\right)^{\prime}= & \hat{f}\left(z_{i}(t)\right)-f(y \cdot t) \\
= & A(y \cdot t)\left(z_{i}(t)-y \cdot t\right)+F\left(z_{i}(t), y \cdot t\right) \\
& -\left(1-\chi_{1}\left(\eta\left(z_{i}\right)\right)\right) \chi_{2}\left(\xi\left(z_{i}\right)\right) \xi\left(z_{i}(t)\right) v\left(z_{i}(t)\right),
\end{aligned}
$$

where $F(z, y)=f(z)-f(y)-A(y)(z-y)$, we have

$$
w^{\prime}=A(y \cdot t) w+B(y \cdot t) w+O\left(\epsilon_{0} w\right),
$$

where

$$
\begin{aligned}
B(y \cdot t) w= & \left\langle\nabla\left(\chi_{1}(\eta(\bar{z}))\right), w\right\rangle \chi_{2}(\xi(\bar{z})) \xi(\bar{z}) v(\bar{z}) \\
& -\left(1-\chi_{1}(\eta(\bar{z}))\right)\left\langle\nabla\left(\chi_{2}\right)(\xi(\bar{z})), w\right\rangle \xi(\bar{z}) v(\bar{z}) \\
& \left.-\left(1-\chi_{1}(\eta(\bar{z}))\right) \chi_{2}(\xi(\bar{z}))\right)\langle\nabla \xi(\bar{z}), w\rangle v(\bar{z}) \\
& -\left(1-\chi_{1}(\eta(\bar{z}))\right) \chi_{2}(\xi(\bar{z})) \xi(\bar{z}) J v(\bar{z}) w
\end{aligned}
$$

with $\bar{z}(t)=\lambda(t) z_{2}(t)+(1-\lambda(t)) z_{1}(t)$ for some $\lambda(t) \in[0,1]$.

Since, for $i=s, c, u$,

$$
\begin{aligned}
& \left(P_{i}(y \cdot t) \Phi(y, t)\right)^{\prime}=\left(P_{i}(y \cdot t)\right)^{\prime} \Phi(y, t)+P_{i}(y \cdot t) A(y \cdot t) \Phi(y, t), \\
& \left(\Phi(y, t) P_{i}(y)\right)^{\prime}=A(y \cdot t) \Phi(y, t) P_{i}(y)=A(y \cdot t) P_{i}(y \cdot t) \Phi(y, t),
\end{aligned}
$$

we have

$$
\left(P_{i}(y \cdot t)\right)^{\prime}=A(y \cdot t) P_{i}(y \cdot t)-P_{i}(y \cdot t) A(y \cdot t) .
$$

By (7),

$$
\begin{aligned}
\left(P_{i}(y \cdot t) w\right)^{\prime} & =A(y \cdot t) P_{i}(y \cdot t) w-P_{i}(y \cdot t) A(y \cdot t) w+P_{i}(y \cdot t) w^{\prime} \\
& =A(y \cdot t) P_{i}(y \cdot t) w+P_{i}(y \cdot t) B(y \cdot t) w+O\left(\epsilon_{0} P_{i}(y \cdot t) w\right) .
\end{aligned}
$$

Lemma 5. Let $w, B$ be as above. Then, for $T>0$, there is a $C(T)>0$ such that

$$
\begin{aligned}
\left|P_{s}(y \cdot t) B(y \cdot t) w(t)\right| & \leq C(T) \epsilon_{0}|w(t)|, \\
\left|B(y \cdot t) P_{s}(y \cdot t) w(t)\right| & \leq C(T) \epsilon_{0}|w(t)|, \\
\left|P_{c u}(y \cdot t) B(y \cdot t) w(t)\right| & \leq C(T)|w(t)|
\end{aligned}
$$

for all $y \in Y$ and $t \in[0, T]$.

Proof. We only prove (9). Let $y \in Y, t \in[0, T]$ and let $w=w(t), \bar{z}=\bar{z}(t)$ be as above. We note that

$$
\begin{aligned}
& \left|P_{s}(y \cdot t) B(y \cdot t) w(t)\right| \leq\left|\left\langle\nabla\left(\chi_{1}(\eta(\bar{z}))\right), w\right\rangle \chi_{2}(\xi(\bar{z})) \xi(\bar{z}) P_{s} v(\bar{z})\right| \\
& +\mid\left(1-\chi_{1}(\eta(\bar{z}))\left\langle\nabla\left(\chi_{2}(\xi(\bar{z}))\right), w\right\rangle \xi(\bar{z}) P_{s} v(\bar{z}) \mid\right. \\
& +\left|\left(1-\chi_{1}(\eta(\bar{z}))\right) \chi_{2}(\xi(\bar{z}))\langle\nabla \xi(\bar{z}), w\rangle P_{s} v(\bar{z})\right| \\
& +\left|\left(1-\chi_{1}(\eta(\bar{z}))\right) \chi_{2}(\xi(\bar{z})) \xi(\bar{z}) P_{s} J v(\bar{z}) w\right| .
\end{aligned}
$$

By Lemma 1 and the definitions of $\chi_{1}, \chi_{2}$, there is a $C>0$ such that $|\nabla \xi(\bar{z})| \leq C$, $\left|\nabla\left(\chi_{1}(\eta(\bar{z}))\right)\right| \leq 4 \epsilon_{0}^{-1},\left|\chi_{2}(\xi(\bar{z})) \xi(\bar{z})\right| \leq C \epsilon_{0},|\nabla \eta(\bar{z})-v(\bar{z})| \leq C \epsilon_{0},\left|\nabla \chi_{2}(\xi(\bar{z}))\right| \leq C$, $|\xi(\bar{z})| \leq C \epsilon_{0}$, and $\left|P_{s} J v(\bar{z}) v_{j}(\bar{z})\right| \leq C$ for $j=1, \cdots, n$. Since $v(\bar{z})$ is tangent to $\hat{M}_{c u}(Y)$, we also have $\left|P_{s} v(\bar{z})\right| \leq C_{0}(T) \epsilon_{0}$ for a constant $C_{0}(T)>0$. It follows 
that there is a constant $C_{1}(T)>0$ such that all terms above are bounded by $C_{1}(T) \epsilon_{0}|w(t)|$.

Proof of Proposition 1. Below, for simplicity, we denote all constants which depend only on $T$ as $C(T)$. Let $w, B$ be as above and let $t \in[0, T], y=Q\left(p_{1}\right)$.

To prove (a), let $\tilde{z}(t)$ be such that

$$
\left|q_{2}(t)-q_{1}(t)\right|=\left|\pi_{s} z_{2}(t)-\pi_{s} z_{1}(t)\right|=\left|D \pi_{s}(\tilde{z}(t))\left(z_{2}(t)-z_{1}(t)\right)\right| .
$$

By Lemma $3|\tilde{z}(t)-y \cdot t| \leq C(T) \epsilon_{0}$. Therefore,

$$
\begin{aligned}
\left|q_{2}(t)-q_{1}(t)\right| & =\left|\pi_{s} z_{2}(t)-\pi_{s} z_{1}(t)\right| \\
& \leq\left|P_{s}(y \cdot t) w(t)\right|+C(T) \epsilon_{0}|w(t)| .
\end{aligned}
$$

Applying the variation of constant formula to (8), we have

$$
\begin{aligned}
P_{s}(y \cdot t) w(t)= & \Phi(y, t) P_{s}(y) w(0) \\
& +\int_{0}^{t} \Phi(y, t) \Phi^{-1}(y, s) P_{s}(y \cdot s) B(y \cdot t) w(s) d s \\
& +\int_{0}^{t} \Phi(y, t) \Phi^{-1}(y, s) O\left(\epsilon_{0} P_{s}(y \cdot s) w(s)\right) d s .
\end{aligned}
$$

By (9),

$$
\begin{aligned}
\left|P_{s}(y \cdot t) w(t)\right| \leq & e^{\alpha t}\left|P_{s}(y) w(0)\right|+\int_{0}^{t} e^{\alpha(t-s)} C(T) \epsilon_{0}\left|P_{s}(y \cdot s) w(s)\right| d s \\
& +\int_{0}^{t} e^{\alpha(t-s)} C(T) \epsilon_{0}\left|P_{c u}(y \cdot s) w(s)\right| d s .
\end{aligned}
$$

It follows from Lemma 2 that

$$
\left|P_{s}(y \cdot t) w(t)\right| \leq e^{\alpha t+C(T) \epsilon_{0}}\left(\left|P_{s}(y) w(0)\right|+C(T) \epsilon_{0} \int_{0}^{t} e^{\alpha(t-s)}\left|P_{c u}(y \cdot s) w(s)\right| d s\right) .
$$

Similarly,

$$
\left|P_{c u}(y \cdot t) w(t)\right| \leq e^{\gamma t+C(T)}\left(\left|P_{c u}(y) w(0)\right|+C(T) \int_{0}^{t} e^{\alpha(t-s)}\left|P_{s}(y \cdot s) w(s)\right| d s\right),
$$

where $\gamma>0$ is such that $\left|\Phi\left(y_{*}, t\right)\right| \leq C e^{\gamma t}\left(y_{*} \in Y\right)$ for some $C>0$.

Substituting (15) into (14) and applying Lemma 2 we have

$$
\left|P_{s}(y \cdot t) w(t)\right| \leq e^{\alpha t+C(T) \epsilon_{0}}\left(\left|P_{s}(y) w(0)\right|+C(T) \epsilon_{0}\left|P_{c u}(y) w(0)\right|\right) .
$$

Substituting (16) into (15) and applying Lemma 2 again, we have

$$
\left|P_{c u}(y \cdot t) w(t)\right| \leq e^{\gamma t+C(T) \epsilon_{0}}\left|P_{c u}(y) w(0)\right|+C(T)\left|P_{s}(y) w(0)\right| .
$$

Finally, applying (11), (16) and (17) to (13), we conclude that

$$
\left|q_{2}(T)-q_{1}(T)\right| \leq\left(e^{C(T) \epsilon_{0}}\left|\Phi_{s}(y, T)\right|+C(T) \epsilon_{0}\right)\left|q_{2}-q_{1}\right|+C(T) \epsilon_{0}\left|p_{2}-p_{1}\right| .
$$

This completes the proof of (a). 
Next we prove (b). Similar to the proof of (a), we have

$$
\begin{aligned}
\left|p_{2}(t)-p_{1}(t)\right| & =\left|\pi_{c u} z_{2}(t)-\pi_{c u} z_{1}(t)\right| \\
& =\left|D \pi_{c u}(\tilde{z}(t))\left(z_{2}(t)-z_{1}(t)\right)\right| \\
& \geq\left|P_{c u}(y \cdot t) w(t)\right|-C(T) \epsilon_{0}|w(t)|,
\end{aligned}
$$

where $\tilde{z}(t)$ is such that $|\tilde{z}(t)-y \cdot t| \leq C(T) \epsilon_{0}$. It remains to estimate $\left|P_{c u}(y \cdot t) w(t)\right|$.

By (7), (9) and (10),

$$
\begin{aligned}
\left(P_{c u}(y \cdot t) w\right)^{\prime}= & A(y \cdot t) P_{c u}(y \cdot t) w+P_{c u}(y \cdot t) B(y \cdot t) w+O\left(\epsilon_{0} P_{c u}(y \cdot t) w\right) \\
= & (A(y \cdot t)+B(y \cdot t)) P_{c u}(y \cdot t) w+P_{c u}(y \cdot t) B(y \cdot t) P_{s}(y \cdot t) w \\
& -P_{s}(y \cdot t) B(y \cdot t) P_{c u}(y \cdot t) w+O\left(\epsilon_{0} P_{c u}(y \cdot t) w\right) \\
= & (A(y \cdot t)+B(y \cdot t)) P_{c u}(y \cdot t) w \\
& +O\left(\epsilon_{0} P_{c u}(y \cdot t) w\right)+O\left(\epsilon_{0} P_{s}(y \cdot t) w\right) .
\end{aligned}
$$

A direct computation yields

$$
\begin{aligned}
\left(\frac{1}{2}\left|P_{c u}(y \cdot t) w\right|^{2}\right)^{\prime}= & \left\langle A(y \cdot t) P_{c u}(y \cdot t) w, P_{c u}(y \cdot t) w\right\rangle \\
& +\left\langle B(y \cdot t) P_{c u}(y \cdot t) w, P_{c u}(y \cdot t) w\right\rangle \\
& +O\left(\epsilon_{0}\left|P_{c u}(y \cdot t) w\right|^{2}\right) .
\end{aligned}
$$

We claim that the following inequality holds:

$$
\begin{aligned}
\left(\frac{1}{2}\left|P_{c u}(y \cdot t) w\right|^{2}\right)^{\prime} \geq & \min \left\{\left\langle A(y \cdot t) P_{c u}(y \cdot t) w, P_{c u}(y \cdot t) w\right\rangle, 0\right\} \\
& +O\left(\epsilon_{0}\left|P_{c u}(y \cdot t) w\right|^{2}\right)
\end{aligned}
$$

Let $\bar{z}=\bar{z}(t)$ be as in (8).

Case 1: $\xi(\bar{z})=0$.

In this case,

$$
\begin{aligned}
& \left\langle B(y \cdot t) P_{c u}(y \cdot t) w, P_{c u}(y \cdot t) w\right\rangle \\
& \quad=-\left(1-\chi_{1}(\eta(\bar{z}))\right)\left\langle\nabla \xi(\bar{z}), P_{c u}(y \cdot t) w\right\rangle\left\langle P_{c u} v(\bar{z}), P_{c u} w\right\rangle .
\end{aligned}
$$

By expressing $P_{c u}(y \cdot t) w$ as a linear combination of $P_{c u}(y \cdot t) v(\bar{z})$ and its orthogonal complement, we see that either (i) $P_{c u}(y \cdot t) w \perp P_{c u}(y \cdot t) v(\bar{z})$ or (ii) $P_{c u} w=$ $\lambda P_{c u} v$ for some $\lambda$.

If (i) holds, then $\left\langle B(y \cdot t) P_{c u} w, P_{c u} w\right\rangle=0$ and (20) is clearly true.

If (ii) holds, then by (ㅁ)

$$
\begin{aligned}
\left\langle\nabla \xi(\bar{z}), P_{c u} w\right\rangle & =\left\langle A^{\top}(\bar{z}) v(\bar{z}), P_{c u} w\right\rangle+\sum_{j=2}^{n} \xi_{j}(\bar{z})\left\langle\left(J v_{j}(\bar{z})\right)^{\top} v(\bar{z}), P_{c u} w\right\rangle \\
& =\left\langle A(\bar{z}) P_{c u} w, v(\bar{z})\right\rangle-\sum_{j=2}^{n} \xi_{j}(\bar{z})\left\langle(J v(\bar{z}))^{\top} v_{j}(\bar{z}), P_{c u} w\right\rangle,
\end{aligned}
$$

where $J v(\bar{z}) P_{c u} w=\lambda J v(\bar{z}) P_{c u} v(\bar{z})=O\left(\epsilon_{0}\right)$. It follows that

$$
\begin{aligned}
& \left\langle(A(y \cdot t)+B(y \cdot t)) P_{c u}(y \cdot t) w, P_{c u}(y \cdot t) w\right\rangle \\
& =\chi_{1}(\eta(\bar{z}))\left\langle A(y \cdot t) P_{c u}(y \cdot t) w, P_{c u}(y \cdot t) w\right\rangle+O\left(\epsilon_{0}\left|P_{c u}(y \cdot t) w\right|^{2}\right) .
\end{aligned}
$$


If $\left\langle A(y \cdot t) P_{c u}(y \cdot t) w, P_{c u}(y \cdot t) w\right\rangle>0$, then the first term above is nonnegative. If $\left\langle A(y \cdot t) P_{c u}(y \cdot t) w, P_{c u}(y \cdot t) w\right\rangle \leq 0$, then the first term above is greater than or equal to $\left\langle A(y \cdot t) P_{c u} w, P_{c u} w\right\rangle$. Hence, (20) holds.

Case 2: $\xi(\bar{z})>0$.

In this case, $\chi_{2}(\xi(\bar{z}))=1$. Therefore,

$$
\begin{aligned}
\left\langle B(y \cdot t) P_{c u}(y \cdot t) w, P_{c u}(y \cdot t) w\right\rangle= & \left\langle\nabla\left(\chi_{1}(\eta(\bar{z}))\right), P_{c u} w\right\rangle \xi(\bar{z})\left\langle P_{c u} w, P_{c u} v(\bar{z})\right\rangle \\
& -\left(1-\chi_{1}(\eta(\bar{z}))\right)\left\langle\nabla \xi(\bar{z}), P_{c u} w\right\rangle\left\langle P_{c u} w, P_{c u} v(\bar{z})\right\rangle \\
& -\left(1-\chi_{1}(\eta(\bar{z}))\right) \xi(\bar{z})\left\langle P_{c u} J v(\bar{z}) P_{c u} w, P_{c u} w\right\rangle \\
& +\left\langle P_{c u}(y \cdot t) J F(\bar{z}, y \cdot t) P_{c u} w, P_{c u} w\right\rangle .
\end{aligned}
$$

By Lemma 1 and the fact that $\nabla\left(\chi_{1}(\eta(\bar{z}))\right)=\chi_{1}^{\prime}(\eta(\bar{z})) \nabla \eta(\bar{z})$, the first term above is a sum of a nonnegative term and a term of order $\epsilon_{0}$. The second term above is of order $\epsilon_{0}$ as in (ii) above. By Lemma 1)(i), the third term above is positive. The last term is of higher order in $\epsilon_{0}$. Therefore,

$$
\begin{aligned}
& \left\langle(A(y \cdot t)+B(y \cdot t)) P_{c u}(y \cdot t) w, P_{c u}(y \cdot t) w\right\rangle \\
& \quad \geq \min \left\{\left\langle A(y \cdot t) P_{c u}(y \cdot t) w, P_{c u}(y \cdot t) w\right\rangle, 0\right\}+O\left(\epsilon_{0}\left|P_{c u}(y \cdot t) w\right|^{2}\right),
\end{aligned}
$$

that is, (20) holds.

Case 3: $\xi(\bar{z})<0$.

In this case, $\left|\chi_{2}(\xi(\bar{z})) \xi(\bar{z})\right|=O\left(\epsilon_{0}^{2}\right)$. Therefore,

$$
\begin{aligned}
& \left|\left\langle\nabla\left(\chi_{1}(\eta(\bar{z}))\right), P_{c u} w\right\rangle \chi_{2}(\xi(\bar{z})) \xi(\bar{z})\left\langle P_{c u}(y \cdot t) v(\bar{z}), P_{c u} w\right\rangle\right| \\
& \quad \leq C(T)\left(\epsilon_{0}\right)^{-1} \epsilon_{0}^{2}\left|P_{c u} w\right|^{2}=C(T) \epsilon_{0}\left|P_{c u} w\right|^{2},
\end{aligned}
$$

and

$$
\begin{aligned}
& \left|\left(1-\chi_{1}(\eta(\bar{z}))\right) \chi_{2}(\xi(\bar{z})) \xi(\bar{z})\left\langle P_{c u}(y \cdot t) J v(\bar{z}) P_{c u} w, P_{c u} w\right\rangle\right| \\
& \leq C(T) \epsilon_{0}^{2}\left(\epsilon_{0}\right)^{-1}\left|P_{c u} w\right|^{2}=C(T) \epsilon_{0}\left|P_{c u} w\right|^{2} .
\end{aligned}
$$

Thus,

$$
\begin{aligned}
& \left\langle(A(y \cdot t)+B(y \cdot t)) P_{c u}(y \cdot t) w, P_{c u}(y \cdot t) w\right\rangle \\
& \quad=\left\langle A(y \cdot t) P_{c u}(y \cdot t) w, P_{c u}(y \cdot t) w\right\rangle+O\left(\epsilon_{0}\left|P_{c u}(y \cdot t) w\right|^{2}\right) .
\end{aligned}
$$

Therefore, (201) holds in any case.

Now let $\theta(t), C(t)$ be nonnegative continuous functions on $t \in[0, T]$ such that

$$
\begin{aligned}
\left(\frac{1}{2}\left|P_{c u} w\right|^{2}\right)^{\prime}= & \min \left\{\left\langle A(y \cdot t) P_{c u}(y \cdot t) w, P_{c u}(y \cdot t) w\right\rangle, 0\right\} \\
& +\left(\theta(t)+C(t) \epsilon_{0}\right)\left|P_{c u}(y \cdot t) w\right|^{2} .
\end{aligned}
$$

Denote

$$
\mathcal{I}=\left\{t \in[0, T]:\left\langle A(y \cdot t) P_{c u} w(t), P_{c u} w(t)\right\rangle<0\right\}
$$

and $\mathcal{I}^{c}=[0, T] \backslash \mathcal{I}$. Since $\mathcal{I}$ is open, we can express $\mathcal{I}$ as a countable union of nonoverlapping intervals, say, $\mathcal{I}=\bigcup_{j=1}^{+\infty}\left(\underline{t}_{j}, \bar{t}^{j}\right)$. It can be verified directly that, for any $t \in\left(\underline{t}_{j}, \bar{t}^{j}\right)$,

$$
\exp \left(\int_{\underline{t}_{j}}^{t}\left(\theta(\tau)+C(\tau) \epsilon_{0}\right) d \tau\right) \Phi\left(y \cdot \underline{t}_{j}, t-\underline{t}_{j}\right) P_{c u}\left(y \cdot \underline{t}_{j}\right) w\left(\underline{t}_{j}\right)
$$


is a solution of (21). Since $P_{c u}(y \cdot t) w(t)$ is also a solution, we have

$$
\begin{aligned}
& \left|P_{c u}(y \cdot t) w(t)\right| \\
& \quad \geq \exp \left(\int_{\underline{t}_{j}}^{t}\left(\theta(\tau)+C(\tau) \epsilon_{0}\right) d \tau\right)\left|\Phi\left(y \cdot \underline{t}_{j}, t-\underline{t}_{j}\right) P_{c u}\left(y \cdot \underline{t}_{j}\right) w\left(\underline{t}_{j}\right)\right| \\
& \quad \geq e^{C(t) \epsilon_{0}}\left|\Phi\left(y \cdot \underline{t}_{j}, t-\underline{t}_{j}\right) P_{c u}\left(y \cdot \underline{t}_{j}\right) w\left(\underline{t}_{j}\right)\right| \\
& \quad \geq e^{C(t) \epsilon_{0}} m\left(\Phi_{c u}\left(y \cdot \underline{t}_{j}, t-\underline{t}_{j}\right)\right)\left|w\left(\underline{t}_{j}\right)\right| .
\end{aligned}
$$

By (21), for $[s, t] \in \mathcal{I}^{c}$, we have

$$
\left|P_{c u}(y \cdot t) w(t)\right| \geq e^{O(\epsilon)(t-s)}\left|P_{c u}(y \cdot s) w(s)\right| .
$$

Applying (22) and (23), and also using (H2) and Lemma 1, we have

$$
\begin{aligned}
\left|P_{c u}(y \cdot t) w(t)\right| \geq & e^{C(T) \epsilon_{0}} m\left(\Phi_{c u}\left(y \cdot \underline{t}_{n}, t-\underline{t}_{n}\right)\right) \cdots m\left(\Phi_{c u}\left(y, \underline{t}_{1}\right)\right)\left|p_{2}-p_{1}\right| \\
& -C(T) \epsilon_{0}\left(\left|q_{2}-q_{1}\right|+\left|p_{2}-p_{1}\right|\right) \\
\geq & e^{C(T) \epsilon_{0}}|| \Phi_{s}\left(y \cdot \underline{t}_{n}, t-\underline{t}_{n}\right)||^{\beta} \cdots|| \Phi_{s}\left(y, t_{1}\right)||^{\beta}\left|p_{2}-p_{1}\right| \\
& -C(T) \epsilon_{0}\left(\left|q_{2}-q_{1}\right|+\left|p_{2}-p_{1}\right|\right) \\
\geq & \left(\left.e^{C(T) \epsilon_{0}}|| \Phi_{s}(y, t)\right|^{\beta}-C(t) \epsilon_{0}\right)\left|p_{2}-p_{1}\right|-C(T) \epsilon_{0}\left|q_{2}-q_{1}\right| .
\end{aligned}
$$

This completes the proof.

\section{Proof of MAIN THEOREM}

5.1. Existence of center-unstable manifold. Using the bundle structure constructed in Section 3, we first choose a function space to which the graph transform will apply.

Define

$$
\Gamma:=\left\{h: U_{\epsilon_{0}} \rightarrow N_{\epsilon_{0}} ; h(p) \in S_{\epsilon_{0}}(p), \forall p \in U_{\epsilon_{0}},|h|_{C^{0}}<\infty\right\}
$$

and

$$
\Gamma_{\rho}:=\{h \in \Gamma: \operatorname{Lip}(h) \leq \rho\},
$$

where $\rho>0$,

$$
\operatorname{Lip}(h)=\sup _{p \in U_{\epsilon_{0}}} \operatorname{Lip}_{p}(h), \text { and } \operatorname{Lip}_{p}(h)=\limsup _{p^{\prime} \rightarrow p, p^{\prime} \in U_{\epsilon_{0}}} \frac{\left|h\left(p^{\prime}\right)-h(p)\right|}{\left|p^{\prime}-p\right|} .
$$

Lemma 6. $\Gamma_{\rho}$ is closed in $\Gamma$.

Proof. Suppose $h_{n} \in \Gamma_{\rho}$ and $h_{n} \rightarrow h$ in $C^{0}$-norm. We shall show that $\operatorname{Lip}_{p}(h) \leq \rho$ for any $p \in U_{\epsilon_{0}}$. For $p^{\prime}, p^{\prime \prime} \in U_{\epsilon_{0}}$, let $\tilde{d}\left(p^{\prime}, p^{\prime \prime}\right)$ denote the induced Euclidean distance between $p^{\prime}$ and $p^{\prime \prime}$ in $U_{\epsilon_{0}}$ (i.e., the length of the shortest path in $U_{\epsilon_{0}}$ joining $p^{\prime}$ and $\left.p^{\prime \prime}\right)$. Then for any $\epsilon>0$, there exists $\delta>0$ such that

$$
(1-\epsilon) \tilde{d}\left(p^{\prime}, p^{\prime \prime}\right) \leq\left|p^{\prime}-p^{\prime \prime}\right| \leq \tilde{d}\left(p^{\prime}, p^{\prime \prime}\right)
$$

whenever $\left|p^{\prime}-p^{\prime \prime}\right| \leq \delta$.

For any $p_{1} \in U_{\epsilon_{0}}$ with $\left|p_{1}-p\right| \leq \delta$, we let $\gamma$ be the shortest path in $U_{\epsilon_{0}}$ from $p_{1}$ to $p$. Then for any $p^{\prime} \in \gamma$, there exists $\delta\left(p^{\prime}\right)>0$ such that

$$
\left|h_{n}\left(p^{\prime \prime}\right)-h_{n}\left(p^{\prime}\right)\right| \leq(\rho+\epsilon)\left|p^{\prime \prime}-p^{\prime}\right|
$$


for all $n$ and $p^{\prime \prime} \in \gamma$ with $\left|p^{\prime \prime}-p^{\prime}\right| \leq \delta\left(p^{\prime}\right)$. Since $\bigcup_{p^{\prime} \in \gamma}\left(B_{\delta\left(p^{\prime}\right) / 2}\left(p^{\prime}\right) \cap \gamma\right)$ covers $\gamma$, there exist

$$
p_{2}, p_{3}, \cdots, p_{m}=p \in \gamma,
$$

ordered from $p_{1}$ to $p$, such that $\bigcup_{i=1}^{m}\left(B_{\delta\left(p_{i}\right) / 2}\left(p_{i}\right) \cap \gamma\right)$ covers $\gamma$. By choosing $\delta$ sufficiently small, we may assume that

$$
p_{i} \notin B_{\delta\left(p_{i+2}\right) / 2}\left(p_{i+2}\right) \cup B_{\delta\left(p_{i-2}\right) / 2}\left(p_{i-2}\right) \text { for } i=3, \cdots, m-2 .
$$

Thus,

$$
\left|p_{i}-p_{i+1}\right|<\delta\left(p_{i}\right) / 2+\delta\left(p_{i+1}\right) / 2<\max \left\{\delta\left(p_{i}\right), \delta\left(p_{i+1}\right)\right\},
$$

which implies that either $p_{i} \in B_{\delta\left(p_{i+1}\right)}\left(p_{i+1}\right)$ or $p_{i+1} \in B_{\delta\left(p_{i}\right)}\left(p_{i}\right)(i=1, \cdots, m-1)$. In any case,

$$
\left|h_{n}\left(p_{i}\right)-h_{n}\left(p_{i+1}\right)\right| \leq(\rho+\epsilon)\left|p_{i}-p_{i+1}\right|
$$

for all $n$ and $i=1, \cdots, m-1$. Therefore,

$$
\begin{aligned}
\left|h_{n}\left(p_{1}\right)-h_{n}(p)\right| & \leq \sum_{i=1}^{m}\left|h_{n}\left(p_{i}\right)-h_{n}\left(p_{i+1}\right)\right| \\
& \leq(\rho+\epsilon) \sum_{i=1}^{m}\left|p_{i}-p_{i+1}\right| \\
& \leq(\rho+\epsilon) \sum_{i=1}^{m} \tilde{d}\left(p_{i}, p_{i+1}\right) \\
& =(\rho+\epsilon) \tilde{d}\left(p_{1}, p\right) \\
& \leq \frac{\rho+\epsilon}{1-\epsilon}\left|p_{1}-p\right| .
\end{aligned}
$$

By taking $n \rightarrow+\infty$ in the above, we have

$$
\left|h\left(p_{1}\right)-h(p)\right| \leq \frac{\rho+\epsilon}{1-\epsilon}\left|p_{1}-p\right| .
$$

Since $\epsilon$ is arbitrary, $\operatorname{Lip}_{p}(h) \leq \rho$.

For each $h \in \Gamma_{\rho}$, the graph, $\operatorname{graph}(h):=\left\{(p, h(p)): p \in U_{\epsilon_{0}}\right\}$, of $h$ is a section of the fiber bundle $N_{\epsilon_{0}}$ with base space $U_{\epsilon_{0}}$. We now define the graph transform.

Let $\Gamma_{\rho}(0<\rho \leq 1)$ be as in (24) for a prescribed $\epsilon_{0}>0$ and let $\phi^{T}(T>0)$ denote the time $T$ map of the modified flow (3). Define the graph transform

$$
\phi^{*}: \Gamma_{\rho} \rightarrow \Gamma_{\rho} \text { by } \phi^{*} h=H, \text { where } \operatorname{graph}(H)=\phi^{T}(\operatorname{graph}(h)) \cap N_{\epsilon_{0}} .
$$

Remark 3. Let $\sigma_{h}(p)=\pi_{c u} \cdot \phi^{T} \cdot(i d, h)(p)$ for $p \in U_{\epsilon_{0}}$. Then $\phi^{*}(h)=\pi_{s} \cdot \phi^{T}$. $(i d, h) \cdot \sigma_{h}^{-1}$, provided that $\sigma_{h}$ maps $U_{\epsilon_{0}}$ to itself and is invertible.

The existence of a center-unstable manifold for the modified system (3) is an immediate consequence of the following proposition.

Proposition 2. Fix $0<\rho \leq 1$. There exist $T>0, \epsilon_{0}=\epsilon_{0}(T)>0$ such that the following holds.

(i) $\phi^{*}: \Gamma_{\rho} \rightarrow \Gamma_{\rho}$ is well-defined.

(ii) $\phi^{*}$ is a contraction mapping with respect to the $C^{0}$-norm.

(iii) Let $h \in \Gamma_{\rho}$ be the fixed point of $\phi^{*}$. Then $M_{c u}(Y):=\operatorname{graph}(h)$ is an invariant Lipschitz manifold of (31). 
Proof. First, by (H2), we can fix a $T>0$ such that

$$
\left\|\Phi_{s}(y, T)\right\|^{1-\beta}<\frac{1}{16}, \quad\left\|\Phi_{s}(y, T)\right\|^{\beta}<\frac{1}{8} .
$$

Let $C(T)$ be as in Proposition 1 We choose an $\epsilon_{0}=\epsilon_{0}(T)$ such that

$$
0<\epsilon_{0}<\frac{\left\|\Phi_{s}(y, T)\right\|^{\beta}}{2 C(T)} .
$$

Let $h \in \Gamma_{\rho}$. We wish to show that $\phi^{*} h \in \Gamma_{\rho}$; that is, $\phi^{T}(\operatorname{graph}(h)) \cap N_{\epsilon_{0}}$ is a function $H$ with $\operatorname{Lip}(H) \leq \rho$. Equivalently, we shall show that, for any $\left(p_{0}, q_{0}\right) \in$ $\phi^{T}(\operatorname{graph}(h)) \cap N_{\epsilon_{0}}$, there exists a $\delta>0$ such that if $(p, q) \in \phi^{T}(\operatorname{graph}(h)) \cap N_{\epsilon_{0}}$ and $\left|(p, q)-\left(p_{0}, q_{0}\right)\right| \leq \delta$, then $\frac{\left|q-q_{0}\right|}{\left|p-p_{0}\right|} \leq \rho$.

Fix a $\left(p_{0}, q_{0}\right) \in \phi^{T}(\operatorname{graph}(h)) \cap N_{\epsilon_{0}}$ and define $\left(p^{*}, q^{*}\right)=\phi^{-T}(p, q)$ for $(p, q) \in$ $\phi^{T}(\operatorname{graph}(h)) \cap N_{\epsilon_{0}}$. Clearly, $p^{*}, q^{*}$ are continuous functions of $(p, q)$. It follows that there exists a $\delta>0$ such that if $\left|(p, q)-\left(p_{0}, q_{0}\right)\right| \leq \delta$, then $\left|\left(p^{*}, q^{*}\right)-\left(p_{0}^{*}, q_{0}^{*}\right)\right| \leq \epsilon_{0}$. Now let $(p, q) \in \phi^{T}(\operatorname{graph}(h)) \cap N_{\epsilon_{0}}$ with $\left|(p, q)-\left(p_{0}, q_{0}\right)\right| \leq \delta$ and denote $\left(p_{1}, q_{1}\right)=$ $\left(p_{0}^{*}, q_{0}^{*}\right),\left(p_{2}, q_{2}\right)=\left(p^{*}, q^{*}\right)$. Due to the overflowing property of the modified flow, we have $\phi^{t}\left(p_{i}, q_{i}\right) \in N_{\epsilon_{0}}(i=1,2)$ for all $0 \leq t \leq T$. By (44) and (15),

$$
\begin{aligned}
\frac{\left|q-q_{0}\right|}{\left|p-p_{0}\right|} & =\frac{\left|q_{2}(T)-q_{1}(T)\right|}{\left|p_{2}(T)-p_{1}(T)\right|} \\
& \leq \frac{\left(e^{C(T) \epsilon_{0}}|| \Phi_{s}(y, T)||+C(T) \epsilon_{0}\right)\left|q_{2}-q_{1}\right|+C(T) \epsilon_{0}\left|p_{2}-p_{1}\right|}{\left(e^{C(T) \epsilon_{0}}|| \Phi_{s}(y, T)||^{\beta}-C(T) \epsilon_{0}\right)\left|p_{2}-p_{1}\right|-C(T) \epsilon_{0}\left|q_{2}-q_{1}\right|} \\
& \leq\left(e^{C(T) \epsilon_{0}}|| \Phi_{s}(y, T)||^{1-\beta}+C(T) \epsilon_{0}\right) \rho \leq \rho .
\end{aligned}
$$

This proves (i).

We now prove (ii). Let $h_{1}, h_{2} \in \Gamma_{\rho}$ and denote $H_{i}=\phi^{*}\left(h_{i}\right)$, for $i=1,2$. For any $p \in U_{\epsilon_{0}}$, we let $p_{1}, p_{2} \in U_{\epsilon_{0}}$ be such that $\pi_{c u} \cdot \phi^{T}\left(p_{i}, h_{i}\left(p_{i}\right)\right)=p(i=1,2)$. By Proposition 1, $\left|p_{2}-p_{1}\right|=O\left(\epsilon_{0}\right)$. Denote $y=Q\left(p_{1}\right)$. Then,

$$
\begin{aligned}
\left|H_{2}(p)-H_{1}(p)\right|= & \left|\pi_{s} \phi^{T}\left(p_{2}, h_{2}\left(p_{2}\right)\right)-\pi_{s} \phi^{T}\left(p_{1}, h_{1}\left(p_{1}\right)\right)\right| \\
\leq & \left|\pi_{s} \phi^{T}\left(p_{1}, h_{1}\left(p_{1}\right)\right)-\pi_{s} \phi^{T}\left(p_{1}, h_{2}\left(p_{1}\right)\right)\right| \\
& +\left|\pi_{s} \phi^{T}\left(p_{1}, h_{2}\left(p_{1}\right)\right)-\pi_{s} \phi^{T}\left(p_{2}, h_{2}\left(p_{2}\right)\right)\right| .
\end{aligned}
$$

By (4),

$$
\begin{aligned}
& \left|\pi_{s} \phi^{T}\left(p_{1}, h_{1}\left(p_{1}\right)\right)-\pi_{s} \phi^{T}\left(p_{1}, h_{2}\left(p_{1}\right)\right)\right| \\
& \quad \leq\left(e^{C(T) \epsilon_{0}}|| \Phi_{s}(y, T)||+C(T) \epsilon_{0}\right)\left|h_{2}\left(p_{1}\right)-h_{1}\left(p_{1}\right)\right| \\
& \quad \leq\left(e^{C(T) \epsilon_{0}}|| \Phi_{s}(y, T)||+C(T) \epsilon_{0}\right)\left|h_{2}-h_{1}\right|
\end{aligned}
$$

and

$$
\begin{aligned}
& \left|\pi_{s} \phi^{T}\left(p_{1}, h_{2}\left(p_{1}\right)\right)-\pi_{s} \phi^{T}\left(p_{2}, h_{2}\left(p_{2}\right)\right)\right| \\
& \quad \leq\left(e^{C(T) \epsilon_{0}}|| \Phi_{s}(y, T)||+C(T) \epsilon_{0}\right)\left|h_{2}\left(p_{1}\right)-h_{2}\left(p_{2}\right)\right|+C(T) \epsilon_{0}\left|p_{2}-p_{1}\right| \\
& \quad \leq\left(e^{C(T) \epsilon_{0}}|| \Phi_{s}(y, T)|| \rho+C(T) \epsilon_{0}\right)\left|p_{2}-p_{1}\right| .
\end{aligned}
$$


Since

$$
\begin{gathered}
\pi_{c u} \phi^{T}\left(p_{2}, h_{2}\left(p_{2}\right)\right)-\pi_{c u} \phi^{T}\left(p_{1}, h_{2}\left(p_{1}\right)\right)+\pi_{c u} \phi^{T}\left(p_{1}, h_{2}\left(p_{1}\right)\right)-\pi_{c u} \phi^{T}\left(p_{1}, h_{1}\left(p_{1}\right)\right) \\
=\pi_{c u} \phi^{T}\left(p_{2}, h_{2}\left(p_{2}\right)\right)-\pi_{c u} \phi^{T}\left(p_{1}, h_{1}\left(p_{1}\right)\right)=0,
\end{gathered}
$$

we have

$$
\left|\pi_{c u} \phi^{T}\left(p_{2}, h_{2}\left(p_{2}\right)\right)-\pi_{c u} \phi^{T}\left(p_{1}, h_{2}\left(p_{1}\right)\right)\right|=\left|\pi_{c u} \phi^{T}\left(p_{1}, h_{2}\left(p_{1}\right)\right)-\pi_{c u} \phi^{T}\left(p_{1}, h_{1}\left(p_{1}\right)\right)\right| .
$$

By (5),

$$
\begin{aligned}
\mid \pi_{c u} \phi^{T}\left(p_{2}, h_{2}\left(p_{2}\right)\right) & -\pi_{c u} \phi^{T}\left(p_{1}, h_{2}\left(p_{1}\right)\right) \mid \\
& \geq\left(e^{C(T) \epsilon_{0}}|| \Phi_{s}(y, T)||^{\beta}-C(T) \epsilon_{0}\right)\left|p_{2}-p_{1}\right| .
\end{aligned}
$$

By (28), (29) and (5),

$$
\begin{aligned}
\left|p_{2}-p_{1}\right| & \leq \frac{\left|\pi_{c u} \phi^{T}\left(p_{2}, h_{2}\left(p_{2}\right)\right)-\pi_{c u} \phi^{T}\left(p_{1}, h_{2}\left(p_{1}\right)\right)\right|}{e^{C(T) \epsilon_{0}}|| \Phi_{s}(y, T)||^{\beta}-C(T) \epsilon_{0}} \\
& =\frac{\left|\pi_{c u} \phi^{T}\left(p_{1}, h_{2}\left(p_{1}\right)\right)-\pi_{c u} \phi^{T}\left(p_{1}, h_{1}\left(p_{1}\right)\right)\right|}{e^{C(T) \epsilon_{0}}|| \Phi_{s}(y, T)||^{\beta}-C(T) \epsilon_{0}} \\
& \leq \frac{C(T) \epsilon_{0}\left|h_{2}\left(p_{1}\right)-h_{1}\left(p_{1}\right)\right|}{e^{C(T) \epsilon_{0}}\left\|\Phi_{s}(y, T)\right\|^{\beta}-C(T) \epsilon_{0}} .
\end{aligned}
$$

Substituting (26), (27) and (30) into (25), we see that

$$
\begin{aligned}
\left|H_{2}(p)-H_{1}(p)\right| \leq & \left(e^{C(T) \epsilon_{0}}|| \Phi_{s}(y, T) \|+C(T) \epsilon_{0}\right) \\
& \cdot\left(1+\frac{C(T) \epsilon_{0}}{e^{C(T) \epsilon_{0}}\left\|\Phi_{s}(y, T)\right\|^{\beta}-C(T) \epsilon_{0}}\right)\left|h_{2}-h_{1}\right| .
\end{aligned}
$$

Let $\lambda$ denote the coefficient of $\left|h_{2}-h_{1}\right|$ in the above. By our choice of $T$ and $\epsilon_{0}$, it is clear that $0<\lambda<1$. This proves (ii).

To prove (iii), we fix a small $\tau_{0}>0$ such that $\phi^{\tau} M_{c u}(Y)$ is a graph for $0 \leq \tau \leq \tau_{0}$. Since

$$
\phi^{T}\left(\phi^{\tau} M_{c u}(Y)\right)=\phi^{\tau}\left(\phi^{T} M_{c u}(Y)\right)=\phi^{\tau} M_{c u}(Y)
$$

by the uniqueness of the fixed point of $\phi^{*}, \phi^{\tau} M_{c u}(Y)=M_{c u}(Y)$ for $0 \leq \tau \leq \tau_{0}$. Now for any $t$, we write $t=\left[\frac{t}{\tau_{0}}\right]+\tau$, where $0 \leq \tau<\tau_{0}$. Then

$$
\phi^{t} M_{c u}(Y)=\phi^{\left[\frac{t}{\tau_{0}}\right]+\tau} M_{c u}(Y)=\phi^{\tau}\left(\phi^{\left[\frac{t}{\tau_{0}}\right]} M_{c u}(Y)\right)=\phi^{\tau} M_{c u}(Y)=M_{c u}(Y),
$$

that is, $M_{c u}(Y)$ is invariant to (3)).

Hereafter, $M_{c u}(Y)$ will be referred to as a center-unstable manifold of $Y$.

5.2. Smoothness of center-unstable manifold. We now discuss the smoothness of $M_{c u}(Y)$ following the ideas of [18], [26], [47]. Namely, we first show that $M_{c u}(Y)$ is $C^{1}$ following the arguments of [18] and then use the $C^{r}$ Section Theorem in [26], 47] to improve the smoothness inductively. For other approaches to prove the smoothness of an invariant manifold (for example, the application of Henry's Lemma and the use of a scale of Banach spaces), we refer the readers to [13, 41, 53, 51, 556.

Recall that, for $p \in U_{\epsilon_{0}}$,

$$
h\left(\sigma_{h}(p)\right)=\pi_{s} \cdot \phi^{T} \cdot(i d, h)(p),
$$

where $\sigma_{h}=\pi_{c u} \cdot \phi^{T} \cdot(i d, h)$. 
Let $D_{p}$ denote the differential operator with respect to $p$. Formally, we obtain

$$
D_{p} h\left(\sigma_{h}(p)\right) D_{p} \sigma_{h}(p)=D_{z}\left(\pi_{s} \phi^{T}\right)(p, h(p))\left(i d, D_{p} h\right)(p),
$$

or

$$
D_{p} h\left(\sigma_{h}(p)\right)=D_{z}\left(\pi_{s} \phi^{T}\right)(p, h(p))\left(i d, D_{p} h\right)(p)\left[D_{p}\left(\pi_{c u} \phi^{T}\right)(p, h(p))\left(i d, D_{p} h\right)(p)\right]^{-1} .
$$

Motivated by the above formula, we define $\mathcal{L}_{h}: T_{p} U_{\epsilon_{0}} \rightarrow T_{\left(\sigma_{h}(p), h\left(\sigma_{h}(p)\right)\right)} S_{\epsilon_{0}}\left(\sigma_{h}(p)\right)$ as

$$
\left(\mathcal{L}_{h} l\right)\left(\sigma_{h}(p)\right)=D_{z}\left(\pi_{s} \phi^{T}\right)(p, h(p))(i d, l(p))\left[D_{p}\left(\pi_{c u} \phi^{T}\right)(p, h(p))(i d, l(p))\right]^{-1} .
$$

Let $E$ be the vector bundle over $U_{\epsilon_{0}}$ for which the fiber at $p \in U_{\epsilon_{0}}$ is the space of bounded linear maps from $T_{p} U_{\epsilon_{0}}$ to $T_{(p, h(p))} S_{\epsilon_{0}}(p)$. Define the bundle map $F_{h}: E \rightarrow E:$

$$
(p, l(p)) \rightarrow\left(\sigma_{h}(p),\left(\mathcal{L}_{h} l\right)\left(\sigma_{h}(p)\right)\right)
$$

Then the following diagram

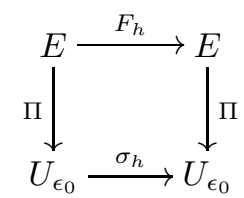

commutes. Denote $L_{0}$ as the zero section of $E$ (that is, $L_{0}: U_{\epsilon_{0}} \rightarrow E$ is such that $L_{0}(p)=0$ for all $\left.p \in U_{\epsilon_{0}}\right)$ and let $L_{n+1}(p)=\mathcal{L}_{h} L_{n}(p), n=0,1, \cdots$. Define the norm of a section $L$ by $|L|=\sup _{p \in U_{\epsilon_{0}}}|L(p)|$ where $|L(p)|$ is the operator norm of $L(p)$.

Lemma 7. $\left|L_{n}\right| \leq \frac{1}{5}$ for all $n \geq 0$.

Proof. Clearly, $\left|L_{0}\right|=0 \leq \frac{1}{5}$. Assume $\left|L_{n}\right| \leq \frac{1}{5}$ for some $n>0$. If $T$ and $\epsilon_{0}$ are as in Proposition 1, then

$$
\begin{aligned}
& \left|L_{n+1}\left(\sigma_{h}(p)\right)\right|=\left|\left(\mathcal{L}_{h} L_{n}\right)\left(\sigma_{h}(p)\right)\right| \\
& \quad \leq\left|D_{z}\left(\pi_{s} \phi^{T}\right)(p, h(p))\left(i d, L_{n}(p)\right)\right|\left|D_{p}\left(\pi_{c u} \phi^{T}\right)(p, h(p))\left(i d, L_{n}(p)\right)\right|^{-1} \\
& \quad \leq \frac{\left(1+\frac{1}{5}\right)\left(e^{C(T) \epsilon_{0}}|| \Phi_{s}(y, T)||+C(T) \epsilon_{0}\right)}{\left(1-\frac{1}{5}\right)\left(e^{C(T) \epsilon_{0}} \|\left.\Phi_{s}(y, T)\right|^{\beta}-C(T) \epsilon_{0}\right)} \leq \frac{1}{5}
\end{aligned}
$$

for all $p \in U_{\epsilon_{0}}$. The proof is then complete by induction.

Lemma 8. $\left\{L_{n}\right\}_{n=0}^{+\infty}$ is a Cauchy sequence.

Proof. Let $p \in U_{\epsilon_{0}}$. For each $n=1,2, \cdots$, we have

$$
\begin{aligned}
L_{n+1} & \left(\sigma_{h}(p)\right)-L_{n}\left(\sigma_{h}(p)\right)=\left(\mathcal{L}_{h} L_{n}\right)(p)-\left(\mathcal{L}_{h} L_{n-1}\right)(p) \\
= & D_{z}\left(\pi_{s} \phi^{T}\right)(p, h(p))\left(i d, L_{n}(p)\right)\left[D_{p}\left(\pi_{c u} \phi^{T}\right)(p, h(p))\left(i d, L_{n}(p)\right)\right]^{-1} \\
& -D_{z}\left(\pi_{s} \phi^{T}\right)(p, h(p))\left(i d, L_{n-1}(p)\right)\left[D_{p}\left(\pi_{c u} \phi^{T}\right)(p, h(p))\left(i d, L_{n-1}(p)\right)\right]^{-1} \\
= & D_{z}\left(\pi_{s} \phi^{T}\right)(p, h(p))\left(0, L_{n}(p)-L_{n-1}(p)\right)\left[D_{p}\left(\pi_{c u} \phi^{T}\right)(p, h(p))\left(i d, L_{n}(p)\right)\right]^{-1} \\
& +D_{z}\left(\pi_{s} \phi^{T}\right)(p, h(p))\left(i d, L_{n-1}(p)\right)\left(\left[D_{p}\left(\pi_{c u} \phi^{T}\right)(p, h(p))\left(i d, L_{n}(p)\right)\right]^{-1}\right. \\
& \left.-\left[D_{p}\left(\pi_{c u} \phi^{T}\right)(p, h(p))\left(i d, L_{n-1}(p)\right)\right]^{-1}\right) .
\end{aligned}
$$


Let $y=Q(p)$. Then

$$
\begin{aligned}
\mid D_{z} & \left(\pi_{s} \phi^{T}\right)(p, h(p))\left(0, L_{n}(p)-L_{n-1}(p)\right)\left[D_{p}\left(\pi_{c u} \phi^{T}\right)(p, h(p))\left(i d, L_{n}(p)\right)\right]^{-1} \mid \\
\leq & \left|D_{z}\left(\pi_{s} \phi^{T}\right)(p, h(p))\right|\left|L_{n}(p)-L_{n-1}(p)\right|\left|D_{p}\left(\pi_{c u} \phi^{T}\right)(p, h(p))\left(i d, L_{n}(p)\right)\right|^{-1} \\
\leq & \left(e^{C(T) \epsilon_{0}}|| \Phi_{s}(y, T)||+C(T) \epsilon_{0}\right)\left(e^{C(T) \epsilon_{0}}|| \Phi_{s}(y, T)||^{\beta}-C(T) \epsilon_{0}\right)^{-1} \\
& \cdot\left(1-\frac{1}{5}\right)^{-1}\left|L_{n}(p)-L_{n-1}(p)\right| \\
\leq & \rho\left(1-\frac{1}{5}\right)^{-1}\left|L_{n}(p)-L_{n-1}(p)\right|,
\end{aligned}
$$

and

$$
\begin{aligned}
& \left|\left[D_{p}\left(\pi_{c u} \phi^{T}\right)(p, h(p))\left(i d, L_{n}(p)\right)\right]^{-1}-\left[D_{p}\left(\pi_{c u} \phi^{T}\right)(p, h(p))\left(i d, L_{n-1}(p)\right)\right]^{-1}\right| \\
& \quad \leq\left|D_{p}\left(\pi_{c u} \phi^{T}\right)(p, h(p))\right|^{-1}\left|\left(i d, L_{n}(p)\right)^{-1}-\left(i d, L_{n-1}(p)\right)^{-1}\right| \\
& \quad \leq\left|D_{p}\left(\pi_{c u} \phi^{T}\right)(p, h(p))\right|^{-1}\left|\left(i d, L_{n}(p)\right)\right|^{-1}\left|L_{n}(p)-L_{n-1}(p)\right|\left|\left(i d, L_{n-1}(p)\right)\right|^{-1} \\
& \quad \leq\left(e^{C(T) \epsilon_{0}}|| \Phi_{s}(y, T)||^{\beta}-C(T) \epsilon_{0}\right)^{-1}\left(1-\frac{1}{5}\right)^{-2}\left|L_{n}(p)-L_{n-1}(p)\right| .
\end{aligned}
$$

Therefore,

$$
\left|L_{n+1}-L_{n}\right| \leq \frac{5}{16} \rho\left|L_{n}-L_{n-1}\right| \leq \frac{5}{16}\left|L_{n}-L_{n-1}\right|,
$$

and $\left\{L_{n}\right\}$ is a Cauchy sequence.

Lemma 9. $h$ is $C^{1}$ and $D_{p} h=L^{1}$, where $L^{1}=\lim _{n \rightarrow \infty} L_{n}$ and $p \in U_{\epsilon_{0}}$.

Proof. We need to show that

$$
\left|h\left(p^{\prime}\right)-h(p)-L^{1}(p) v\left(p^{\prime}, p\right)\right|=o\left(\left|p^{\prime}-p\right|\right),
$$

as $p^{\prime} \rightarrow p$ in $U_{\epsilon_{0}}$, where $\exp _{p} v\left(p^{\prime}, p\right)=p^{\prime}$. We note that $p^{\prime}-p$ is not necessarily in $T_{p} U_{\epsilon_{0}}$, but $p^{\prime}-p-v\left(p^{\prime}, p\right)=O\left(\left|p^{\prime}-p\right|^{2}\right)$ (see [50]).

Following [18], define an increasing function $\gamma:(0,1) \rightarrow \mathbb{R}$ by

$$
\gamma(a)=\sup _{0<\left|p^{\prime}-p\right|<a} \frac{\left|h\left(p^{\prime}\right)-h(p)-L^{1}(p) v\left(p^{\prime}, p\right)\right|}{\left|p^{\prime}-p\right|} .
$$

Then it suffices to show that $\gamma(a) \rightarrow 0$ as $a \rightarrow 0$.

Note that

$$
\begin{aligned}
h\left(\sigma_{h}\left(p^{\prime}\right)\right)-h\left(\sigma_{h}(p)\right)= & \pi_{s} \phi^{T}\left(p^{\prime}, h\left(p^{\prime}\right)\right)-\pi_{s} \phi^{T}(p, h(p)) \\
= & D_{z}\left(\pi_{s} \phi^{T}\right)(p, h(p))\left(p^{\prime}-p, h\left(p^{\prime}\right)-h(p)\right) \\
& +O\left(\left|p^{\prime}-p\right|^{2}\right),
\end{aligned}
$$

and

$$
\begin{aligned}
\sigma_{h}\left(p^{\prime}\right)-\sigma_{h}(p)= & \pi_{c u} \phi^{T}\left(p^{\prime}, h\left(p^{\prime}\right)\right)-\pi_{c u} \phi^{T}(p, h(p)) \\
= & D_{p}\left(\pi_{c u} \phi^{T}\right)(p, h(p))\left(p^{\prime}-p, h\left(p^{\prime}\right)-h(p)\right) \\
& +O\left(\left|p^{\prime}-p\right|^{2}\right) .
\end{aligned}
$$

Since

$$
L^{1}\left(\sigma_{h}(p)\right)=D_{z}\left(\pi_{s} \phi^{T}\right)(p, h(p))\left(i d, L^{1}(p)\right)\left[D_{p}\left(\pi_{c u} \phi^{T}\right)(p, h(p))\left(i d, L^{1}(p)\right)\right]^{-1},
$$

we have

$$
L^{1}\left(\sigma_{h}(p)\right) v\left(\sigma_{h}\left(p^{\prime}\right), \sigma_{h}(p)\right)=D_{z}\left(\pi \phi^{T}\right)(p, h(p))\left(i d, L^{1}(p)\right) v\left(p^{\prime}, p\right)
$$


and

$$
\begin{aligned}
& \left|h\left(\sigma_{h}\left(p^{\prime}\right)\right)-h\left(\sigma_{h}(p)\right)-L^{1}\left(\sigma_{h}(p)\right) v\left(\sigma_{h}\left(p^{\prime}\right), \sigma_{h}(p)\right)\right| \\
& =\left|D_{z}\left(\pi_{s} \phi^{T}\right)(p, h(p))\left(p^{\prime}-p-v\left(p^{\prime}, p\right), h\left(p^{\prime}\right)-h(p)-L^{1}(p) v\left(p^{\prime}, p\right)\right)\right| \\
& \quad+O\left(\left|p^{\prime}-p\right|^{2}\right) \\
& \leq\left(e^{C(T) \epsilon_{0}}|| \Phi_{s}(y, T)||+C(T) \epsilon_{0}\right)\left|h\left(p^{\prime}\right)-h(p)-L^{1}(p) v\left(p^{\prime}, p\right)\right| \\
& \quad+O\left(\left|p^{\prime}-p\right|^{2}\right) .
\end{aligned}
$$

But by Proposition 1

$$
\operatorname{Lip}_{p}\left(\sigma_{h}\right) \geq e^{C(T) \epsilon_{0}}\left\|\Phi_{s}(y, T)\right\|^{\beta}-C(T) \epsilon_{0} .
$$

Therefore,

$$
\begin{aligned}
& \frac{\left|h\left(\sigma_{h}\left(p^{\prime}\right)\right)-h\left(\sigma_{h}(p)\right)-L^{1}\left(\sigma_{h}(p)\right) v\left(\sigma_{h}\left(p^{\prime}\right), \sigma_{h}(p)\right)\right|}{\left|\sigma_{h}\left(p^{\prime}\right)-\sigma_{h}(p)\right|} \\
& \leq\left(e^{C(T) \epsilon_{0}}|| \Phi_{s}(y, T)||+C(T) \epsilon_{0}\right) \frac{\left|h\left(p^{\prime}\right)-h(p)-L^{1}(p) v\left(p^{\prime}, p\right)\right|+O\left(\left|p^{\prime}-p\right|^{2}\right)}{\left|\sigma_{h}\left(p^{\prime}\right)-\sigma_{h}(p)\right|} \\
& \leq \frac{e^{C(T) \epsilon_{0}}|| \Phi_{s}(y, T)||+C(T) \epsilon_{0}}{e^{C(T) \epsilon_{0}}|| \Phi_{s}(y, T)||^{\beta}-C(T) \epsilon_{0}} \frac{\left|h\left(p^{\prime}\right)-h(p)-L^{1}(p) v\left(p^{\prime}, p\right)\right|}{\left|p^{\prime}-p\right|}+O\left(\left|p^{\prime}-p\right|\right) \\
& \leq \rho \frac{\left|h\left(p^{\prime}\right)-h(p)-L^{1}(p) v\left(p^{\prime}, p\right)\right|}{\left|p^{\prime}-p\right|}+O\left(\left|p^{\prime}-p\right|\right) .
\end{aligned}
$$

That is, there are $\delta>0$ and $\beta(a)=O(a)$ such that

$$
\gamma(a) \leq \rho \gamma(\delta a)+\beta(a) .
$$

Now, if $\delta \leq 1$, then $\gamma(a) \leq(1-\rho)^{-1} \beta(a) \rightarrow 0$ as $a \rightarrow 0$. If $\delta>1$, by (30), then

$$
\begin{aligned}
\gamma\left(a \delta^{-m}\right) & \leq \rho^{m} \gamma(a)+\beta\left(a \delta^{-m}\right)+\cdots+\rho^{m-1} \beta\left(a \delta^{-1}\right) \\
& \leq \rho^{m} \gamma(a)+(1-\rho)^{-1} \beta\left(a \delta^{-1}\right) .
\end{aligned}
$$

It follows that $\gamma(a) \rightarrow 0$ as $a \rightarrow 0$. This completes the proof.

The following lemma is an immediate consequence of the general $C^{r}$ Section Theorem (see [26], 47]).

Lemma 10. Let $B$ be the disc bundle of radius $\delta$ in E. Suppose $\mathcal{L}_{h}: B_{p} \rightarrow B_{\sigma_{h}(p)}$ is Lipschitz with constant $k, \sigma_{h}$ and $F$ are $C^{s}, D^{j} F$ and $D^{j} \sigma_{h}^{-1}$ are bounded for $1 \leq j \leq s$. If $k \mu^{s}<1$, where $\mu=\operatorname{Lip}\left(\sigma_{h}^{-1}\right)$, then $L^{1}$ is $C^{s}$; hence $h$ is $C^{s+1}$.

Proposition 3. There exists $\epsilon_{0}$ such that, if $h \in \Gamma_{\rho}$ is the fixed point of $\phi^{*}$ in Proposition 0 , then $h$ is $C^{d}$, where $d$ is the integer defined in Theorem 1 .

Proof. By Lemma $9 h$ is $C^{1}$. Now assume that $h$ is $C^{s}$, for $s \leq d-1$. Since $d \leq r$, $\sigma_{h}$ and $F$ are $C^{s}$. Let $T$ and $\epsilon_{0}(T)$ be as in Proposition $\square$ and let $\epsilon_{0} \leq \epsilon_{0}(T)$. We 
then have

$$
k \leq \sup _{y \in Y}\left\{\frac{e^{C(T) \epsilon_{0}}\left\|\Phi_{s}(y, T)\right\|+C(T) \epsilon_{0}}{e^{C(T) \epsilon_{0}}\left\|\Phi_{s}(y, T)\right\|^{\beta}-C(T) \epsilon_{0}}\right\}
$$

and

$$
\mu \leq \sup _{y \in Y}\left(e^{C(T) \epsilon_{0}}\left\|\Phi_{s}(y, T)\right\|^{\beta}-C(T) \epsilon_{0}\right)^{-1} .
$$

Hence

$$
k \mu^{s} \leq \frac{e^{C(T) \epsilon_{0}}\left\|\Phi_{s}(y, T)\right\|+C(T) \epsilon_{0}}{\left(e^{C(T) \epsilon_{0}}\left\|\Phi_{s}(y, T)\right\|^{\beta}-C(T) \epsilon_{0}\right)^{s+1}} .
$$

The right-hand side above approaches to $\|\Phi(y, T)\|^{1-\beta(s+1)}$ as $\epsilon_{0} \rightarrow 0$, which is less than 1 because $s+1 \leq d<\frac{1}{\beta}$. So, if we choose $\epsilon_{0}$ small (smaller than $\epsilon_{0}(T)$ in general), then $k \mu^{s}<1$. It follows from Lemma 10 that $h$ is $C^{s+1}$. By induction, $h$ is $C^{d}$.

\subsection{Proof of Theorem 1.}

Proof. Proposition 3 implies that there exists a $C^{d}$ center-unstable manifold $M_{c u}(Y)$ of the modified flow (3). Since, in a small neighborhood of $Y, \hat{f}$ agrees with $f$, $M_{c u}(Y)$ is also a locally invariant manifold of (11) and $T_{y} M_{c u}(Y)=V_{c u}(y)$ for $y \in Y$. By reversing the time, we similarly obtain a locally invariant $C^{d}$ manifold $M_{c s}(Y)$ of (11) in a neighborhood of $Y$ with $T_{y} M_{c s}(Y)=V_{c s}(y)$ for $y \in Y$. The intersection $M_{c}(Y)=M_{c u}(Y) \cap M_{c s}(Y)$ therefore gives a locally invariant $C^{d}$ manifold of (1) with $T_{y} M_{c}(Y)=V_{c}(y)$ for $y \in Y$, which is a desired center manifold.

If $g \in C^{r}$ is such that $|g-f|_{C^{r}}$ is small, then so is $|\hat{g}-\hat{f}|_{C^{r}}$, where $\hat{g}$ is the modification of $g$ in the same fashion as that of $f$. By arguments of [18] and [6], there exists a locally invariant $C^{d}$ manifold $M_{c u}(\hat{g})$ for $\hat{g}$ which is $C^{d}$ close to $M_{c u}(Y)$. Moreover, $M_{c u}(\hat{g})$ is clearly a locally invariant manifold for $g$ when restricting to a small neighborhood of $Y$. Similarly, there exists a locally invariant $C^{d}$ manifold $M_{c s}(g)$ for $g$ which is $C^{d}$ close to $M_{c s}(Y)$. The intersection $M_{c}(g)=M_{c u}(g) \cap M_{c s}(g)$ is then a $C^{d}$ invariant manifold for $g$ which is $C^{d}$ close to $M_{c}(Y)$. This shows that $M_{c}(Y)$ is $C^{d}$ persistent.

Clearly, $M_{c u}(Y)$ carries all the solutions locally bounded in negative time, and $M_{c s}(Y)$ carries all the solutions locally bounded in positive time. We then conclude that $M_{c}(Y)$ carries all solutions which are locally bounded in $\mathbb{R}$. The proof of Theorem 1 is now complete.

\section{ACKNowledgments}

We would like to thank Jack Hale for encouragement and for challenging questions. We are grateful to C. Chicone, K. Lu, J. Mallet-Paret, K. Palmer, B. Sandstede for many valuable discussions. We also wish to thank the referees for comments and suggestions which led to significant improvements of the present paper. During the preparation of this paper, the first author was supported by NSF grants DMS 9306265, DMS 9404199 and NIST grant 60NANB2D1276; the last author was supported by NSF grant DMS 9501412 and a Rosenbaum fellowship. All authors were supported in part by the Center for Dynamical Systems and Nonlinear Studies, Georgia Institute of Technology. 


\section{REFERENCES}

[1] Afraimovich, V., Chow, S.-N. and Liu, W. (1995). Lorenz-type attractors from co-dimension one bifurcations. J. Dyn. Diff. Eqn. 7, 375-407. MR 96c:58097]

[2] Afraimovich, V., and Shilnikov, L. (1974). On Some Global Bifurcations Connected with the Disappearance of a Fixed Point of Saddle-node Type. Doklady Akad. Nauk. SSSR 219, 1281-1285 (in Russian). English translation in Sov. Math. Doklady.

[3] Ball, J.M. (1973). Saddle point analysis for an ordinary differential equation in a Banach space and an application to dynamic buckling of a beam. Nonlinear Elasticity (R. W. Dickey, ed.), Academic Press, New York, 93-160. MR 48:3342

[4] Bates, P.W. and Jones, C.K.R.T. (1989). Invariant manifolds for semilinear partial differential equations. Dyn. Reported 2, 1-38, Wiley. MR 90g:58017

[5] Bates, P., Lu, K. and Zeng, C. Existence and persistence of invariant manifolds for semiflows in Banach space. Mem. Amer. Math. Soc. to appear. MR 97:11

[6] Bronstein, I. U. and Kopanskii, A. Ya. Smooth Invariant Manifolds and Normal Forms. World Scientific Series on Nonlinear Science. Series A, 7, 1994. of center manifolds. Proc. Roy. Soc. Edinburgh. 120 A, 61-77. MR 96d:58123

[7] Carr, J. Applications of Center Manifold Theory. Applied Mathematical Sciences, 35, Springer-Verlag, New York, 1981. MR 83g:34039

[8] Chafee, N. (1971). A bifurcation problem for functional differential equations of finitely retarded type. J. Math. Anal. Appl. 35, 312-348. MR 49:10997

[9] Chen, X.-Y., Hale, J. and Tan, B. (1997). Invariant foliations of $C^{1}$ semigroups in Banach spaces. J. Diff. Eqn. 139, 293-318. MR 98m:47109

[10] Chenciner, A. and Iooss, G. (1979). Bifurcations des tores invariants. Arch. Rat. Mech. Anal. 71, 301-306. MR 81c:58049

[11] Chow, S.-N. and Hale, J. Method of Bifurcation Theory. Springer-Verlag, 1982. MR 84e:58019

[12] Chow, S-N., Li, C. and Wang, D. Normal Forms and Bifurcation of Planar Vector Fields. Cambridge University Press, 1994. MR 95i:58161

[13] Chow, S-N. and Lu, K. (1988). $C^{k}$ Center unstable manifolds. Proc. Roy. Soc. Edinburgh. 79, 189-231. MR 90a:58148

[14] Chow, S-N. and Lu, K. (1995). Invariant manifolds and foliations for quasiperiodic systems. J. Diff. Eqn. 117, 1-27. MR 96b:34064

[15] Chow, S-N. and Yi, Y. (1994). Center manifold and stability for skew-product flows. J. Dyn. Diff. Eqn. 6, 543-582. MR 95k:58142

[16] Diliberto, S.P. (1960). Perturbation theorems for periodic surfaces, I. Rend. Circ. Mat. Palermo, Ser. 2, 9, 265-299. MR 26:420a

[17] Dumoritier, F., Roussarie, R., Sotomayor, J. and Zoladek, H. Bifurcations of planar vector fields. Lect. Notes in Math. No. 1480, Springer-Verlag Berlin Heidelberg, 1991. MR 93f:58165

[18] Fenichel, N. (1971). Persistence and smoothness of invariant manifolds for flows. Indiana Univ. Math. J. 21, 193-226. MR 44:4313

[19] Fenichel, N. (1979). Geometric singular perturbation theory for ordinary differential equations. J. Diff. Eqn. 31, 53-98. MR 80m:58032

[20] Foias, C., Sell, G. and Temam, R. (1988). Inertial manifolds for nonlinear evolutionary equations. J. Diff. Eqn. 73, 309-353. MR 89e:58020

[21] Guckenheimer, J. and Holmes, P. Nonlinear Oscillators, Dynamical Systems and Bifurcations of Vector Fields. Springer-Verlag, 1985. MR 85f:58002

[22] Hadamard, J. (1901). Sur Líteration et les solutions asymptotiques des equations differentielles. Bull. Soc. Math. France 29, 224-228.

[23] Hale, J. (1961). Integral manifolds of perturbed differential systems. Ann. Math., 73, 496531. MR 23:A1108

[24] Hale, J. Ordinary Differential Equations. John Wiley, New York, 1969. MR 54:7918

[25] Henry, D. Geometric Theory of Semilinear Parabolic Equations. Lect. Notes in Math. No. 840, Spring-Verlag, Berlin, 1983. MR 83j:35084

[26] Hirsch, M., Pugh, C. and Shub, M. Invariant Manifolds. Lect. Notes in Math. No. 583, Springer-Verlag, New York, 1976. MR 58:18595 
[27] Homburg, A. (1996). Global aspects of homoclinic bifurcations of vector fields. Mem. Amer. Math. Soc. 121, no. 578. MR 96i:58125

[28] Johnson, R.A. (1978). Concerning a theorem of Sell. J. Diff. Eqn. 30, 324-339. MR 80b:34058

[29] Jones, C.K.R.T. and Kopell, N. (1994). Tracking invariant manifolds with differential forms in singularly perturbed systems. J. Diff. Eqn. 107, 1-25. MR 95c:34085

[30] Kelley, A. (1967). The stable, center-stable, center, center-unstable, unstable manifolds. $J$. Diff. Eqn. 3, 546-570. MR 36:4096

[31] Kurzweil, J. (1968). Invariant manifolds of differential systems. Differencialńye Uravnenija 4 (part 1), 785-797. MR 49:9342

[32] Li, Y., McLaughlin, D., Shatah, J. and Wiggins, S. (1996). Persistent homoclinic orbits for perturbed nonlinear Schrödinger equations. Comm. Pure Appl. Math. 49, 1175-1255. MR 98d:35208

[33] Lyapunov, A.M. (1947). Problème géneral de la stabilité du mouvement. Annals Math. Studies 17, Princeton, N.J. (originally published in Russian, 1892).

[34] Mãné, R. (1977). Persistent manifolds are normally hyperbolic. Trans. Amer. Math. Soc. Vol 246, 261-283. MR 80c:58019

[35] Mielke, A. (1988). Reduction of quasilinear elliptic equations in cylindrical domains with applications. Math. Methods Appl. Soc. 10, 51-66. MR 89d:35063

[36] Nash, J. (1956). The imbedding problem for Riemannian manifolds. Ann. Math. 63, 20-63. MR 17:782b

[37] Palmer, K. (1987). On the stability of the center manifold. J. of Appl. Math. Phys. (ZAMP) 38, 273-278. linearization theorem. J. Math. Anal. Appl. 41, 753-758. MR 89h:34047

[38] Perron, O. (1928). Über stabilität und asymptotisches verhalten der integrale von differentialgleichungssystemen. Math. Z. 29, 129-160.

[39] Pliss, V.A. (1964). Principal reduction in the theory of stability of motion. Izv. Akad. Nauk. SSSR Mat. Ser. 28, 1297-1324. MR 32:7861

[40] Pliss, V.A. and Sell, G. (1996). Approximation dynamics and the stability of invariant sets. IMA Preprint Series \#1393.

[41] Rybakowski, K.P. (1993). An abstract approach to smoothness of invariant manifolds. Applicable Anal. 49, 119-150. MR 95c:58027

[42] Sacker, R. (1965). A perturbation theorem for invariant Riemannian manifolds. Differential Equations and Dynamical Systems, Proc. Symp. Diff. Eqn. Dyn. Syst., 43-54, Academic Press, New York, 1967. MR 36:1784

[43] Sacker, R. and Sell, G. (1978). A spectral theory for linear differential systems. J. Diff. Eqn. 27, 320-358. MR 58:18604

[44] Sakamoto, K. (1994). Estimates on the strength of exponential dichotomies and application to integral manifolds. J. Diff. Eqn. 107, 259-279. MR 95b:34052

[45] Sandstede, B. (1993). Verzweigungstheorie homokliner verdopplungen. Report No. 7, Institut für Angewandte Analysis und Stochastik, Germany.

[46] Selgrade, J. (1975). Isolated invariant sets for flows on vector bundles. Trans. Amer. Math. Soc. 203, 359-390. MR 51:4322

[47] Shub, M. Global Stability of Dynamical Systems. Springer-Verlag, 1986. MR 87m:58086

[48] Sijbrand, J. (1985). Properties of center manifolds. Trans. Amer. Math. Soc. 289, 431-469. MR 86i:58099

[49] Smale, S. (1967). Differentiable Dynamical Systems. Bull. Amer. Math. Soc. 73, 747-817. MR 37:3598

[50] Spivak, M. A Comprehensive Introduction to Differential Geometry, I, II, III Publish or Perish, 1979. MR 42:2369 MR 51:8962 MR 42:6726

[51] Vanderbauwhede, A. (1989). Center manifolds, normal forms and elementary bifurcations. Dyn. Reported, 2, 89-169.

[52] Vanderbauwhede, A. and Iooss, G. (1990). Center manifold theory in infinite dimensions. Dyn. Report. (N. S.) 1, 125-163. MR 93f:58174

[53] Vanderbauwhede, A. and van Gils, S.A. (1987). Center manifolds and contractions on a scale of Banach spaces. J. Funct. Anal. 72, 209-224. IMR 88d:58085

[54] Whitney, H. (1936). Differential manifolds. Ann. Math., (2)37, 645-680. 
[55] Wiggins, S. Normally Hyperbolic Invariant Manifolds in Dynamical Systems. Applied Math. Sciences 105, Springer-Verlag, 1994. MR 95g:58163

[56] Yi, Y. (1993). A generalized integral manifold theorem. J. Diff. Eqn. 102, 153-187. MR 94c:58148

School of Mathematics, Georgia Institute of Technology, Atlanta, Georgia 30332 0190

E-mail address: chow@math.gatech.edu

(Additional address for S.-N. Chow) Department of Mathematics, National University of Singapore, Singapore 119262

Department of Mathematics, University of Missouri-Columbia, Columbia, Missouri 65211

Current address: Department of Mathematics, University of Kansas, Lawrence, Kansas 66045

E-mail address: wliu@math.ukans.edu

School of Mathematics, Georgia Institute of Technology, Atlanta, Georgia 303320190

E-mail address: yi@math.gatech.edu 